\title{
ON SYSTEMS OF COMPLEXITY ONE IN THE PRIMES
}

\author{
KEVIN HENRIOT
}

\begin{abstract}
Consider a translation-invariant system of linear equations $V \mathbf{x}=0$ of complexity one, where $V$ is an integer $r \times t$ matrix. We show that if $A$ is a subset of the primes up to $N$ of density at least $C(\log \log N)^{-1 / 25 t}$, there exists a solution $\mathbf{x} \in A^{t}$ to $V \mathbf{x}=0$ with distinct coordinates. This extends a quantitative result of Helfgott and de Roton for three-term arithmetic progressions, while the qualitative result is known to hold for all translation-invariant systems of finite complexity by the work of Green and Tao.
\end{abstract}

\section{INTRODUCTION}

Consider a matrix $V \in \mathcal{M}_{r \times t}(\mathbb{Z})$ with coefficients on each line summing to 0 , a condition we term translation-invariant. We are interested in special instances of the problem of finding a distinct-coordinates solution $\mathbf{y} \in A^{t}$ to the system of equations $V \mathbf{y}=0$, where $A$ is a dense subset of the set $\mathcal{P}_{N}$ of the primes up to a large integer $N$, and when the relative density decays with $N$. Note that the distinct-coordinates condition excludes trivial solutions of the form $(u, \ldots, u)$, while the conditions of homogeneity and translation-invariance on the system of equations are necessary to expect a Szemeréditype theorem for $V \mathbf{y}=0$, as can be seen by examining the case of a single linear equation (see e.g. [23, Theorem 1.3]).

We may assume that $V$ has rank $r$ up to removing redundant equations. Furthermore, we may work in practice with a parametrization $\psi: \mathbb{Z}^{t-r} \stackrel{\sim}{\rightarrow} \mathbb{Z}^{t} \cap \operatorname{Ker}(V)$, and look instead for occurences of distinct-coordinates values of $\psi$ in $A^{t}$. The canonical setting of study is that of the single translation-invariant equation $y_{1}+y_{3}=2 y_{2}$, which detects 3 -term arithmetic progressions, themselves parametrized by the system of forms

$$
\psi\left(x_{1}, x_{2}\right)=\left(x_{1}, x_{1}+x_{2}, x_{1}+2 x_{2}\right) .
$$

It is then a well-known result of Green [9] that every subset of $\mathcal{P}_{N}$ of positive density contains a non-trivial three-term arithmetic progression; and the extension of this result to progressions of any length is the celebrated Green-Tao theorem [12]. Green's argument [9] actually allowed for densities as low as $(\log \log \log \log N)^{-1 / 2+o(1)}$, and Helfgott and de Roton [14] later obtained a remarkable quantitative strenghtening of this result. 
Theorem 1 (Helfgott, de Roton). Suppose that $A$ is a subset of $\mathcal{P}_{N}$ of density at least ${ }^{1}$

$$
(\log \log N)^{-1 / 3+o(1)} .
$$

Then there exists a non-trivial three-term arithmetic progression in A.

Naslund [20] further improved the lowest admissible density to $(\log \log N)^{-1+o(1)}$. It should be noted that these transference arguments preserve, up to a logarithm, the exponent in the best known bounds for Roth's theorem by Sanders [24], on which they rely: indeed Sanders established that three-term arithmetic progressions may be found in any subset of $[N]$ of density at least $(\log N)^{-1+o(1)}$.

In the context of counting linear patterns in primes [13], Green and Tao introduced the notion of Cauchy-Schwarz complexity ${ }^{2}$ (abbreviated as complexity in the following) for systems of integer linear forms. Precisely, we say that a system of $t$ distinct linear forms $\left(\psi_{1}, \ldots, \psi_{t}\right)$ has complexity at most $s$ when, for every $i \in[t]$, it is possible to partition the set of forms $\left\{\psi_{j}, j \neq i\right\}$ into at most $s+1$ sets, such that $\psi_{i}$ does not belong to the linear span of any of those sets. The condition of finite complexity is then equivalent to requiring that no two forms of the system be linearly dependent. By extension, we define the complexity of a matrix $V$ to be that of any parametrization $\psi: \mathbb{Z}^{d} \rightarrow \mathbb{Z}^{t} \cap \operatorname{Ker}(V)$, this property being independent of the choice of $\psi$.

Systems of complexity at most one may be analyzed by methods of classical Fourier analysis, whereas cases of higher complexities require much more involved techniques [6, 11]. We focus on the case of complexity one here, for it is possible to derive strong quantitative bounds in that setting, and for it may provide insight on how to quantify results of higher complexity. On the qualitative side, it is known that a translationinvariant system of equations $V \mathbf{y}=0$ of finite complexity is non-trivially solvable in any subset of the primes of positive upper density: this follows from the Green-Tao theorem [12] on arithmetic progressions in the primes, by a simple folklore argument ${ }^{3}$. Our main finding is that, in the case of complexity one, quantitative bounds of the quality of Helfgott and de Roton's may be achieved.

Theorem 2. Let $V \in \mathcal{M}_{r \times t}(\mathbb{Z})$ be a translation-invariant matrix of rank $r$ and complexity one. There exists a positive constant $C$ depending at most on $r, t, V$ such that, if

\footnotetext{
${ }^{1}$ Throughout this introduction, we write $\left(\log _{k} N\right)^{o(1)}$ for unspecified factors of the form $C\left(\log _{k+1} N\right)^{C}$ with $C>0$, where $\log _{k}$ is the $k$-th iterated logarithm.

${ }^{2}$ A more subtle notion of complexity, called true complexity, was later developed by Gowers and Wolf [7]. However it does not seem, at present, to cover the setting of unbounded prime-counting functions.

${ }^{3}$ Given a system $\psi: \mathbb{Z}^{d} \rightarrow \operatorname{Ker}(V) \cap \mathbb{Z}^{t}$ of finite complexity, pick $u \in \mathbb{Z}$ so that all the values $c_{i}=\psi_{i}(u)$ are distinct. A simple variation of the proof of Green and Tao [12] makes it possible to find a pattern $\left(x+c_{1} d, \ldots, x+c_{t} d\right)$ with distinct coordinates in the primes, and this yields a non-trivial solution of $V \mathbf{y}=0$ since $x+c_{i} d=\psi_{i}(x+d u)$.
} 
$A$ is a subset of $\mathcal{P}_{N}$ of density at least

$$
C(\log \log N)^{-1 / 25 t}
$$

there exists $\mathbf{y} \in A^{t}$ with distinct coordinates such that $V \mathbf{y}=0$.

Our argument also preserves the aforementioned feature of Naslund's refinement of the Helfgott-de Roton transference principle: in the complexity one regime, it converts logarithmic density bounds $(\log N)^{-\gamma}$ for Szemerédi-type theorems in the integers, to doubly logarithmic bounds $(\log \log N)^{-\gamma+\varepsilon}$ for Szemerédi-type theorems in the primes. We mention however that Theorem 2 is surpassed, in certain special cases, by results in the integers. Indeed, an important result of Schoen and Shkredov [25] states that any single translation-invariant equation in a least 6 variables is non-trivially solvable in any subset of $[N]$ of density $e^{-(\log N)^{1 / 6-o(1)}}$, and hence in $\mathcal{P}_{N}$, however it is not clear whether or how that result extends to the case of several equations. Furthermore, in certain "degenerate" cases where the $r \times t$ matrix $V$ may be subdivided into translationinvariant $r \times t_{i}$ submatrices, the system of equations may even be solvable at densities $N^{-c}$ : we refer to the work of Shapira [27], generalizing that of Ruzsa [23], for precise statements.

To motivate Theorem 2, we now give some illustrative examples of systems of complexity one. First, any single translation-invariant equation has complexity one, although in that case a simple modification of the argument of Helfgott and de Roton [14] yields Theorem 2. A more representative example of a system of complexity one is that of " $d$ points and their midpoints", corresponding to the set of equations $\left(y_{i i}+y_{j j}=2 y_{i j}\right)_{1 \leqslant i<j \leqslant d}$, whose solutions over $\mathbb{Q}$ are parametrized, with some multiplicity, by ${ }^{4} \psi(x)=\left(x_{0}+x_{i}+x_{j}\right)_{1 \leqslant i \leqslant j \leqslant d}$. It can be arduous in general to determine whether a system of equations has complexity one: Vinuesa [33] has determined, by an elaborate combinatorial argument, that the system of translation-invariant equations corresponding to magic $n \times n$ squares has complexity one for $n \geqslant 4$. For a more general discussion of the complexity one setting, we refer to Section 4.

Next, we discuss the principal ideas behind the proof of Theorem 2. The main structure of our argument follows the transference principle, introduced by Green [9] and further developped by Green and Tao [12], and by which one lifts a dense subset of the primes to a dense subset of the integers. More precisely, we initially follow the efficient transference strategy of Helfgott and de Roton [14], which builds on that of Green and Tao [10], and we incorporate Naslund's [20] estimates. Denoting by $\lambda_{A}$ the renormalized indicator function of a dense subset $A$ of the primes, we therefore compare the average of $\lambda_{A}$ over $\psi$-patterns to that of a smoothed version $\lambda_{A}^{\prime}$ of itself, which behaves as a dense subset

\footnotetext{
$\overline{{ }^{4} \text { This system }}$ is the linear part of Example 4 from [13, Section 1], composed with a certain surjection.
} 
of the integers of almost the same density. As usual, there is a little technical subtelty in the form of the $W$-trick, by which we consider, instead of the set $A$, its intersection with an arithmetic progression of modulus $W=\prod_{p \leqslant \omega} p$. A critical feature of Helfgott and de Roton's argument [14] is then that it requires a modulus $\omega \sim c \log N$.

At this point we invoke a beautiful recent result of Shao [26], who improved on a first result of Dousse [3], and generalized the logarithmic bounds of Bourgain [1] for Roth's theorem to a model system of complexity one. More precisely, Shao [26] investigated the system $\psi(x)=\left(x_{0}+x_{i}+x_{j}\right)_{1 \leqslant i \leqslant j \leqslant d}$, and proved that a set $A$ of density $(\log N)^{-1 / 6 d(d+1)+o(1)}$ in $[N]$ contains a non-trivial configuration $\psi(x) \in A^{d(d+1) / 2}$. As envisioned by Shao [26, p. 2], his argument naturally extends to general systems of complexity one, at the cost of adressing certain technical complications. The first, and simplest step of our proof is therefore to formally derive this extension, while also keeping track of the number of pattern occurences. Considering $\lambda_{A}^{\prime}$ as a dense set of integers, this extension then shows that $\lambda_{A}^{\prime}$ has a large pattern count.

Provided that we could prove that the difference of pattern counts for $\lambda_{A}$ and $\lambda_{A}^{\prime}$ is small, this would be enough to conclude that the original set $A$ contains many $\psi$ configurations. However, while the count of three-term progressions investigated by Helfgott and de Roton [14] has a simple Fourier expression, which can be controlled by restriction estimates for primes [10], such is not the case in general for systems of complexity one. To address this issue, we bound the difference of pattern counts via the generalized Von Neumann theorem of Green and Tao [13], which in the complexity-one setting asserts that, given functions $f_{1}, \ldots, f_{t}$ on $\mathbb{Z}_{N^{\prime}}$ with $N^{\prime} \sim C N$ majorized by a pseudorandom weight (a notion whose meaning shall be clear shortly), we have

$$
\left|\mathbb{E}_{n \in \mathbb{Z}_{N^{\prime}}^{d}} f_{1}\left(\psi_{1}(n)\right) \ldots f_{t}\left(\psi_{t}(n)\right)\right| \leqslant\left\|f_{i}\right\|_{U^{2}}+o(1)
$$

as $N \rightarrow \infty$. Properly quantified, the method of Green and Tao $[12,13]$ produces a $o(1)$ term of size $(\log N)^{-c}$ in the above, however it requires a small modulus $\omega \sim c \log \log N$, which is too expensive to apply the efficient transference estimates of Helgott and de Roton [14].

To majorize prime-counting functions associated to $W$-tricked primes, Green and Tao use a weight $\nu: \mathbb{Z}_{M} \rightarrow \mathbb{R}^{+}$constructed from a smoothly truncated convolution of the Möbius function, whose averages where first considered by Goldston, Pintz and Yildirim [4]. The $o(1)$-term arising in (1.1) then depends on the level of pseudorandomness of this weight, and the key estimate we establish towards this is the asymptotic

$$
\mathbb{E}_{n \in \mathbb{Z}_{N^{\prime}}^{d}} \nu\left(\theta_{1}(n)\right) \ldots \nu\left(\theta_{t}(n)\right)=1+O_{d, t, \theta}\left(\frac{1}{(\log N)^{1-o(1)}}\right),
$$


valid for every affine system $\theta: \mathbb{Z}_{N^{\prime}}^{d} \rightarrow \mathbb{Z}_{N^{\prime}}^{t}$ of finite complexity and bounded linear part, and for a large modulus $\omega \sim c \log N$. This corresponds to the "linear forms condition" in $[12,13]$, while we do not need the harder-to-quantify "correlation condition" from there in our simpler setting. Equipped with this estimate, we verify that the functions $\lambda_{A}$ and $\lambda_{A}^{\prime}$ used by Helfgott and de Roton are majorized by averaged variants of $\nu$, and we finally apply (1.1) to bound the difference of pattern counts.

Remarks. Very recently, and while we were writing this article, Conlon, Fox and Zhao have completed an exposition of the Green-Tao theorem [2], in which they also revisited Green and Tao's computations on correlations of GPY weights under the assumption of finite complexity. Their number-theoretic computations [2, Section 9] turn out to be quite similar to ours from Section 5, although our argument optimizes certain parameters further.

Acknowledgements. We are grateful to our adviser Régis de la Bretèche for valuable advice on writing. We also wish to thank our friends Crystel Bujold, Dimitri Dias, Oleksiy Klurman, Marzieh Mehdizad for helpful discussions on many topics of number theory. We would further like to thank Pablo Candela, Harald Helfgott, Neil Lyall, Eric Naslund, Hans Parshall and Fernando Shao for interesting discussions on problems related to this paper.

Funding. This work was partially supported by the ANR Caesar ANR-12-BS01-0011.

\section{Overview}

In this section we explain the organization of this paper, and how it relates to the structure of our argument presented in the introduction.

The preliminaries to our argument are contained in Sections 3 and 4 . The little notation we need is introduced in Section 3, while Section 4 is there to gather (almost) all arguments of a linear algebraic nature needed in the article.

With these prequisites in place, the first logical part of our argument is the aforementioned extension of Shao's [26] result, and since it require few new ideas we place it at the end of the article in Appendix A. The bulk of our proof of Theorem 2 is then contained in Sections 5-7. In Section 5, we carry out the computation of correlations of the GPY weights

$$
\Lambda_{\chi, R, W}(n)=\left(\frac{\phi(W)}{W} \log R\right)\left(\sum_{d \mid W n+b} \mu(d) \chi\left(\frac{\log d}{\log R}\right)\right)^{2},
$$

where $W=\prod_{p \leqslant \omega} p$ and $\chi$ is a certain smooth cutoff function. We follow Green and Tao's original computation [13, Appendix D], but we analyze the local Eulor factors involved in more detail, in order to allow for a large modulus $\omega=c \log N$. In Section 6, we construct a pseudorandom weight on $\nu$ over $\mathbb{Z}_{M}$ out of $\Lambda_{\chi, R, W}: \mathbb{Z} \rightarrow \mathbb{R}^{+}$for a larger scale 
$M \sim C N$, taking care to preserve quantitative error terms. We also state a quantitative version of Green and Tao's generalized Von Neumann theorem [13, Appendix C]. In Section 7, we prove Theorem 2, by first lifting the problem to the integers via the transference principle of Helfgott-de Roton [14] and the quantitative generalized Von Neumann theorem obtained earlier, and by then applying the extension of Shao's result derived in Appendix A.

\section{Notation}

We have attempted to respect most current conventions of notation in additive combinatorics [8] throughout, and therefore we keep this section to the bare minimum.

Given an integer $N$, we write $[N]=\{1, \ldots, N\}$. Given reals $x<y$, we also write $[x, y]_{\mathbb{Z}}=\mathbb{Z} \cap[x, y]$, and we let $\mathcal{P}$ denote the set of all primes. Given a property $\mathbf{P}$, we write $1(\mathbf{P})$ for the boolean which equals 1 when $\mathbf{P}$ is true, and 0 otherwise. When $X$ is a set and $\mathbf{P}_{x}$ is a property depending on a variable $x \in X$, we write

$$
\mathbb{P}_{x \in X}\left(\mathbf{P}_{x}\right)=|X|^{-1} \#\left\{x \in X: \mathbf{P}_{x}\right\} .
$$

Given a function $f$ on $X$, we also write $\mathbb{E}_{X} f=\mathbb{E}_{x \in X} f(x)=|X|^{-1} \sum_{x \in X} f(x)$, or simply $\mathbb{E} f$ when the set of averaging is clear from the context.

We make occasional use of Landau's $o, O$-notation and of Vinogradov's asymptotic notations $f \ll g, f \gg g, f \asymp g$. As is common in additive combinatorics, we also let $c$ and $C$ denote positive constants whose value may change at each occurence, and which are typically taken to be respectively very small or very large. Unless otherwise stated, all implicit and explicit constants we introduce are absolute: they do not depend on surrounding parameters.

Finally, we use several local conventions on notation, and therefore we advise the reader to pay close attention to the preamble of each section.

\section{LineAR ALGEBRA PRELIMINARIES}

In this section, we discuss the notion of complexity of systems of linear forms, following the very transparent exposition by Green and Tao in [13, Sections 1 and 4], and by Tao in [29]. We also consider the simple problems of parametrizing the kernel of a matrix corresponding to a system of equations, and of defining an analog notion of complexity for such a matrix.

We consider an integral domain $\mathbb{A}$, together with its field of fractions $\mathbb{K}$; in our article we only ever consider $\mathbb{A}=\mathbb{Z}$ or $\mathbb{A}=\mathbb{Z}_{M}$ with $M$ prime. A linear form over the free module $\mathbb{A}^{d}$ naturally induces one over $\mathbb{K}^{d}$, and accordingly all the linear algebra notions are considered over $\mathbb{K}$. This is somewhat overly formal, however it allows us to define certain notions for linear forms over $\mathbb{Z}$ and $\mathbb{Z}_{M}$ at once. Note that throughout this 
article, we consider systems of linear forms $\psi: \mathbb{A}^{d} \rightarrow \mathbb{A}^{t}$ as formal triples $(\psi, d, t)$ to avoid repeatedly introducing dimension parameters $d, t$.

Definition 1 (Complexity). Consider a system of linear forms $\psi=\left(\psi_{1}, \ldots, \psi_{t}\right)$ : $\mathbb{A}^{d} \rightarrow \mathbb{A}^{t}$. For $i \in[t]$, the complexity of $\psi$ at $i$ is the minimal integer $s \geqslant 0$ for which there exists a partition $[t] \backslash\{i\}=X_{1} \sqcup \cdots \sqcup X_{s+1}$ into non-empty sets such that $\psi_{i} \notin\left\langle\psi_{j}: j \in X_{k}\right\rangle$ for all $k \in[s+1]$, when such an integer exists ${ }^{5}$. Otherwise we set the complexity at $i$ to $\infty$. The complexity of $\psi$ is the maximum of the complexities of $\psi$ at $i$ over all $i \in[t]$.

We also recall the following important observation from [13, Section 1].

Lemma 1. A system of linear forms $\psi=\left(\psi_{1}, \ldots, \psi_{t}\right): \mathbb{A}^{d} \rightarrow \mathbb{A}^{t}$ has finite complexity if and only if no two forms $\psi_{i}, \psi_{j}$ with $i \neq j$ are linearly dependent.

We next recall the standard notion of normal form, and to do so we introduce a slightly non-standard piece of terminology. We say that a linear form $\theta\left(x_{1}, \ldots, x_{d}\right)=$ $a_{1} x_{1}+\cdots+a_{d} x_{d}$ depends on the variable $x_{k}$ when $a_{k} \neq 0$; we do not mean this in an exclusive sense so that the form may also depend on other variables. While that definition may seem mathematically akward, it corresponds to the intuitive way to think about explicit system of forms.

Definition 2 (Normal form). A system of linear forms $\psi=\left(\psi_{1}, \ldots, \psi_{t}\right): \mathbb{A}^{d} \rightarrow \mathbb{A}^{t}$ is in exact $s$-normal form at $i \in[t]$ when there exists a set of indices $J_{i} \subset[d]$ such that $\left|J_{i}\right|=s+1$ and

(i) $\psi_{i}\left(x_{1}, \ldots, x_{d}\right)$ depends on all variables $x_{k}, k \in J_{i}$,

(ii) for all $j \neq i, \psi_{j}\left(x_{1}, \ldots, x_{d}\right)$ does not depend on all variables $x_{k}, k \in J_{i}$.

We say that $\psi$ is in $s$-normal form when it is in exact $s_{i}$-normal form with $s_{i} \leqslant s$ at every $i \in[t]$.

As explained in [13, Section 4], a system $\psi$ in exact $s$-normal form at $i$ has complexity at most $s$ at $i$, and conversely one may always put a system of complexity $s$ in $s$-normal form, up to adding a certain number of "dummy" variables.

Proposition 1 (Normal extension). A system of linear forms $\psi: \mathbb{Z}^{d} \rightarrow \mathbb{Z}^{t}$ of complexity $s$ admits an s-normal extension $\psi^{\prime}: \mathbb{Z}^{d+e} \rightarrow \mathbb{Z}^{t}$ of the form $\psi^{\prime}(x, y)=\psi(x+\varphi(y))$, where $\varphi: \mathbb{Z}^{e} \rightarrow \mathbb{Z}^{d}$ is a linear form.

We will also have the occasion to consider systems of affine-linear forms, often abbreviated as "affine systems" throughout the article. Consistently with [13], we write an

\footnotetext{
${ }^{5}$ In the special (and unimportant) case where $t=1$, we set the complexity at $i=1$ to 0 .
} 
affine system $\psi$ as $\psi=\psi(0)+\dot{\psi}$, where $\dot{\psi}$ is the linear part of $\psi$, and we extend previous definitions by declaring $\psi$ to be of complexity $s$ or in $s$-normal form when its linear part is. We also need to consider reductions of forms modulo a large prime $M$ later on, in which case we need to keep track of the size of the coefficients of the forms involved.

Definition 3 (Form and matrix norms). Suppose that $\psi=\left(\psi_{1}, \ldots, \psi_{t}\right): \mathbb{A}^{d} \rightarrow \mathbb{A}^{t}$ is an affine system, and write $\psi_{i}\left(x_{1}, \ldots, x_{d}\right)=a_{i 1} x_{1}+\cdots+a_{i d} x_{d}+b_{i}$ for every $i \in[t]$. When $\mathbb{A}=\mathbb{Z}$ and $M \geqslant 1$, we define

$$
\|\psi\|_{M}=\sum_{i \in[t]} \sum_{j \in[d]}\left|a_{i j}\right|+\sum_{i \in[t]}\left(\left|b_{i}\right| / M\right)
$$

and we simply write $\|\psi\|$ when all $b_{i}$ are zero. When $\mathbb{A}=\mathbb{Z}_{M}$, we define

$$
\|\psi\|=\sum_{i \in[t]} \sum_{j \in[d]}\left\|a_{i j}\right\|_{\mathbb{T}_{M}}+\sum_{i \in[t]}\left\|b_{i} / M\right\|_{\mathbb{T}}
$$

where $\|\cdot\|_{\mathbb{T}_{L}}=d(\cdot, L \mathbb{Z})$. Finally, for $V=\left[\lambda_{i j}\right] \in \mathcal{M}_{r \times t}(\mathbb{Z})$, we write $\|V\|=\sum_{i, j}\left|\lambda_{i j}\right|$.

We now return to our main topic of interest, that is, translation-invariant equations in the integers. As for systems of forms, we consider matrices $V \in \mathcal{M}_{r \times t}(\mathbb{Z})$ as formal triples $(V, r, t)$.

Definition 4. We say that $V=\left[a_{i j}\right] \in \mathcal{M}_{r \times t}(\mathbb{Z})$ is translation-invariant when

$$
a_{i 1}+\cdots+a_{i t}=0 \quad \forall i \in[r]
$$

Given a matrix $V \in \mathcal{M}_{r \times t}(\mathbb{Z})$ corresponding to a system of equations $V \mathbf{y}=0$, we now define the complexity of $V$ at an indice $i \in[t]$, and its global complexity, to be that of any system of linear forms $\psi: \mathbb{Q}^{d} \rightarrow \operatorname{Ker}(V)$. The following proposition ensures that such a definition does not depend on the choice of parametrization $\psi$.

Proposition 2 (Matrix complexity criterion). Consider a matrix $V \in \mathcal{M}_{r \times t}(\mathbb{Z})$ with lines $L_{1}, \ldots, L_{r}$ and $t \geqslant 2$, and a system of linear forms $\psi: \mathbb{Q}^{d} \rightarrow \operatorname{Ker}(V)$. Then $\psi$ has complexity at most $s_{0}$ at $i \in[t]$ if and only if there exists $0 \leqslant s \leqslant s_{0}$ and a partition $[t] \backslash\{i\}=X_{1} \sqcup \cdots \sqcup X_{s+1}$ into non-empty sets such that, for every $k \in[s+1]$,

$$
\left(e_{i}+\sum_{j \in X_{k}} \mathbb{Q} e_{j}\right) \cap\left\langle{ }^{\mathrm{t}} L_{1}, \ldots,{ }^{\mathrm{t}} L_{r}\right\rangle=\varnothing,
$$

where $\left(e_{i}\right)_{1 \leqslant i \leqslant t}$ is the canonical basis of $\mathbb{Q}^{t}$. 
Proof. Consider $i \in[t]$ and a partition $[t] \backslash\{i\}=X_{1} \sqcup \ldots X_{s+1}$ into non-empty sets. For any $k \in[s+1]$ and $\lambda \in \mathbb{Q}^{X_{k}}$, we have an equivalence

$$
\begin{aligned}
& \psi_{i}+\sum_{j \in X_{k}} \lambda_{j} \psi_{j}=0 \\
\Leftrightarrow & x_{i}+\sum_{j \in X_{k}} \lambda_{j} x_{j}=0 \text { for all } x \in \operatorname{Ker}(V) \\
\Leftrightarrow & e_{i}+\sum_{j \in X_{k}} \lambda_{j} e_{j} \in \operatorname{Ker}(V)^{\perp} .
\end{aligned}
$$

Furthermore, by orthogonality in $\mathbb{Q}^{t}$,

$$
\operatorname{Ker}(V)^{\perp}=\left(\left\langle{ }^{\mathrm{t}} L_{1}, \ldots,{ }^{\mathrm{t}} L_{t}\right\rangle^{\perp}\right)^{\perp}=\left\langle{ }^{\mathrm{t}} L_{1}, \ldots,{ }^{\mathrm{t}} L_{r}\right\rangle .
$$

Therefore $\psi_{i} \in\left\langle\psi_{j}, j \in X_{k}\right\rangle$ if and only if there exists $\lambda \in \mathbb{Q}^{X_{k}}$ such that $e_{i}+\sum_{j} \lambda_{j} e_{j} \in$ $\left\langle{ }^{\mathrm{t}} L_{1}, \ldots,{ }^{\mathrm{t}} L_{r}\right\rangle$. The proposition follows by considering the contrapositive.

We shall have the occasion to work with two standard types of parametrizations for the integer kernel of a translation-invariant matrix. The first is the usual normal form, which is useful when working with primes, while the second has an added shift variable, which is useful for the regularity computations of Appendix A. In both cases, it is critical to work with a base parametrization $\psi$ in normal form, in order to bound averages over patterns $\left(\psi_{1}(n), \ldots, \psi_{t}(n)\right)$ by a certain Gowers norm (see Propositions 3 and 19 below).

Proposition 3 (Kernel parametrization). Suppose that $V \in \mathcal{M}_{r \times t}(\mathbb{Z})$ is a translationinvariant matrix of rank $r$ and complexity at most $s$. Then there exists a linear surjection

$$
\psi: \mathbb{Z}^{d} \rightarrow \mathbb{Z}^{t} \cap \operatorname{Ker}(V)
$$

in s-normal form. An alternate linear surjection is then given by

$$
\varphi: \mathbb{Z}^{d+1} \rightarrow \mathbb{Z}^{t} \cap \operatorname{Ker}(V),
$$

where $\varphi$ is defined by $\varphi_{i}\left(x_{0}, x\right)=x_{0}+\psi_{i}(x)$ for every $i \in[t]$ and $\left(x_{0}, x\right) \in \mathbb{Z} \times \mathbb{Z}^{d}$.

Proof. The set $\mathbb{Z}^{t} \cap \operatorname{Ker}(V)$ is a lattice which is easily seen to be of rank $t-r$ (e.g. by first solving $V \mathbf{y}=0$ over $\mathbb{Q}$, then clearing denominators), so that there exists a linear isomorphism $\psi: \mathbb{Z}^{t-r} \stackrel{\sim}{\longrightarrow} \mathbb{Z}^{t} \cap \operatorname{Ker}(V)$ of complexity at most $s$. Since extensions in the sense of Proposition 1 preserve the image of a form, we may choose an alternate linear parametrization $\psi^{\prime}: \mathbb{Z}^{d} \stackrel{\sim}{\longrightarrow} \mathbb{Z}^{t} \cap \operatorname{Ker}(V)$ in $s$-normal form for a certain $d \geqslant t-r$.

Since the matrix $V$ is translation-invariant, we have $V \mathbf{1}=0$, where $\mathbf{1}=(1, \ldots, 1)$. Therefore we may define another surjection $\varphi: \mathbb{Z} \times \mathbb{Z}^{d} \rightarrow \mathbb{Z}^{t} \cap \operatorname{Ker}(V)$ by $\varphi\left(x_{0}, x\right)=$ $x_{0} \mathbf{1}+\psi^{\prime}(x)$.

Note that a system of linear forms $\psi: \mathbb{Z}^{d} \rightarrow \mathbb{Z}^{t}$ in 1-normal form is, at every position $i \in[t]$, either in exact 0 -normal form or in exact 1 -normal form. In practice we can 
always eliminate the first possibility, and while not of fundamental importance, this fact allows us to simplify our argument in some places.

Proposition 4. Suppose that $V \in \mathcal{M}_{r \times t}(\mathbb{Z})$ is a matrix of complexity one with no zero columns and $t \geqslant 3$, and $\psi: \mathbb{Z}^{d} \rightarrow \mathbb{Z}^{t} \cap \operatorname{Ker}(V)$ is a system of linear forms in 1-normal form. Then $\psi$ is in exact 1-normal form at every $i \in[t]$.

Proof. Let $C_{1}, \ldots, C_{t}$ denote the columns of $V$ and consider an indice $i \in[t]$. By Proposition 2 , the statement that $\psi$ has non-zero complexity at $i$ is equivalent to

$$
\begin{aligned}
\psi_{i} \in\left\langle\psi_{j}, j \neq i\right\rangle & \Leftrightarrow\left(e_{i}+\sum_{j \neq i} \mathbb{Q} e_{j}\right) \cap\left\langle{ }^{\mathrm{t}} L_{1}, \ldots,{ }^{\mathrm{t}} L_{r}\right\rangle \neq \varnothing \\
& \Leftrightarrow \exists \mu \in \mathbb{Q}^{r}: \sum_{j=1}^{r} \mu_{j}{ }^{\mathrm{t}} L_{j} \cdot e_{i}=1 \\
& \Leftrightarrow \exists \mu \in \mathbb{Q}^{r}: \mu \cdot C_{i} \neq 0,
\end{aligned}
$$

and this last condition is satisfied if and only if $C_{i}$ is non-zero. Since $\psi$ may have complexity only zero or one, this concludes the proof under our assumption on the matrix.

By similar orthogonality considerations, one can establish that a matrix has complexity at most one if and only if when any of its columns is excluded, the set of remaining columns may be partitioned into two classes, in a way that the excluded column belongs to the linear span of each class. This provides a concrete criterion, which overlaps strongly with a set of conditions designed by Roth [22] and resurfacing in work of Liu, Spencer and Zhao $[18,19]$, but we do not dwelve on this relationship here. One more simple fact we require about (translation-invariant) systems of equations is a bound on the number of integer solutions with two equal coordinates in a box.

Lemma 2 (Number of degenerate solutions). Suppose that $V \in \mathcal{M}_{r \times t}(\mathbb{Z})$ has rank $r$ and finite complexity, and let $i, j$ be two distinct indices in $[t]$. Then

$$
\#\left\{y \in[-N, N]_{\mathbb{Z}}^{t}: V y=0 \text { and } y_{i}=y_{j}\right\} \ll_{V} N^{t-r-1} \text {. }
$$

Proof. Consider the hyperplane $H=\left\{y \in \mathbb{Q}^{t}: y_{i}=y_{j}\right\}$. The subspace $\operatorname{Ker}(V) \cap H$ of $\mathbb{Q}^{t}$ has dimension less than $t-r-1$, since $\operatorname{Ker}(V)$ is not contained in $H$ : indeed if this were the case, there would exist a parametrization $\psi: \mathbb{Z}^{d} \rightarrow \mathbb{Z}^{t} \cap \operatorname{Ker}(V)$ with $\psi_{i}=\psi_{j}$, contradicting the assumption of finite complexity. The bound then follows by simple linear algebraic considerations.

Finally, we collect together some facts about the preservation of certain properties of affine systems under the operations of reduction modulo $M$ or lifting from $\mathbb{Z}_{M}$ to $\mathbb{Z}$. We omit the proofs, which are accessible by simple linear algebra. 
Fact 1. Suppose that $V \in \mathcal{M}_{r \times t}(\mathbb{Z})$ is a translation-invariant matrix of rank $r$ and $\psi: \mathbb{Z}^{d} \rightarrow \mathbb{Z}^{t} \cap \operatorname{Ker}_{\mathbb{Q}}(V)$ is a system of linear forms in exact $s_{i}$-normal form over $\mathbb{Z}$ at every $i \in[t]$. Provided that $M>\max \left(t !\|\psi\|^{t}, r !\|V\|^{r}\right), \psi$ reduces modulo $M$ to a system of linear forms $\theta: \mathbb{Z}_{M}^{d} \rightarrow \operatorname{Ker}_{\mathbb{Z}_{M}}(V)$ is in exact $s_{i}$-normal form over $\mathbb{Z}_{M}$ at every $i \in[t]$, and such that $\|\theta\|=\|\psi\|$.

Fact 2. Suppose that $\theta: \mathbb{Z}_{M}^{d} \rightarrow \mathbb{Z}_{M}^{t}$ is an affine system of finite complexity over $\mathbb{Z}_{M}$, and $M>2\|\dot{\theta}\|$. Then $\theta$ is the reduction modulo $M$ of an affine system $\psi: \mathbb{Z}^{d} \rightarrow \mathbb{Z}^{t}$ of finite complexity over $\mathbb{Z}$ and such that $\|\psi\|_{M}=\|\theta\|,\|\dot{\psi}\|=\|\dot{\theta}\|$.

\section{Correlations of GPY weights}

The aim of this section is to construct efficient pseudorandom weights over $\mathbb{Z}$ majorizing the measure associated to $W$-tricked primes. The weight we consider (see Definition 6 below) is a truncated divisor sum whose correlations were first investigated by Goldston, Pintz and Yildirim [4,5] in the context of small gaps between primes. Green and Tao $[12,13]$ investigated its pseudorandom behavior in greater generality, and this weight is by now a standard tool, e.g. in the context of detecting polynomial patterns in primes $[17,30,31]$. Throughout this section, we will assume familiarity with [13, Appen$\operatorname{dix} \mathrm{D}]$.

We now fix an integer $N$ larger than some absolute constant, and we let $\omega \geqslant 1$ be a parameter. We also let $W=\prod_{p \leqslant \omega} p$ and we fix an integer $b$ such that $(b, W)=1$. It is useful to have a notation for the normalized indicator function of $W$-tricked primes.

Definition 5 (Measure of $W$-tricked primes). We let

$$
\lambda_{b, W}(n)=\frac{\phi(W)}{W}(\log N) \cdot 1(n \in[N] \text { and } b+W n \in \mathcal{P}) .
$$

Our goal is thus to construct a weight function over $\mathbb{Z}$ majorizing $\lambda_{b, W}$, and satisfying strong pseudorandomness asymptotics. Note that $o(1)$ terms throughout this article are to be understood as $N \rightarrow \infty$, and do not depend on any dimension or any affine system involved.

Proposition 5 (Pseudorandom majorant over $\mathbb{Z}$ ). Let $D \geqslant 1$ be a parameter. There exists a constant $C_{D}$ such that the following holds. For $N \geqslant C_{D}$ and $\omega=c_{0} \log N$, there exists $\nu: \mathbb{Z} \rightarrow \mathbb{R}^{+}$such that, for every $\varepsilon>0$,

$$
0 \leqslant \lambda_{b, W} \ll_{D} \nu \ll_{\varepsilon} N^{\varepsilon}
$$


and, for any $P \geqslant N^{c_{1}}$ and any affine system $\psi: \mathbb{Z}^{d} \rightarrow \mathbb{Z}^{t}$ of finite complexity and such that $d, t,\|\dot{\psi}\| \leqslant D$,

$$
\mathbb{E}_{n \in[P]^{d}} \nu\left[\psi_{1}(n)\right] \ldots \nu\left[\psi_{t}(n)\right]=1+O_{D}\left(\frac{1}{(\log N)^{1-o(1)}}\right) .
$$

Note that simply applying [13, Theorem D.3] would be insufficient for our purpose, since the error there is $e^{O(\sqrt{\omega})}(\log N)^{-1 / 20}$ and therefore it is non-trivial only for $\omega \leqslant$ $c(\log \log N)^{2}$, thus rendering the methods of Helfgott and de Roton [14] unapplicable. The argument of [12] also requires a modulus $\omega \leqslant c \log \log N$. Our construction follows closely that in [13, Appendix D], however with one important difference: we make a stronger assumption of finite complexity on the system of linear forms, and under this assumption we obtain improved estimates on the Euler products involved. We also remark that for the purpose of proving Theorem 2, any error term of the form $(\log N)^{-c}$ in (5.1) would suffice, however we take the opportunity here to determine the highest level of pseudorandomness attainable from Green and Tao's approach.

We let $R=N^{\eta}$, where $\eta$ is a small positive constant specified later on. We consider a family of reals $\rho: \mathbb{N} \rightarrow\left[0, e^{2}\right]$ such that $\rho(1)=1$ and with support on $[R]$, which we also specify later on. Our main object of study in this section is the following expression, which may be seen as a smooth Selberg-type weight.

Definition 6 (GPY weight). We let $h_{R, W}=\frac{\phi(W)}{W} \log R$ and

$$
\Lambda_{\chi, R, W}(n)=h_{R, W}\left(\sum_{m \mid W n+b} \mu(m) \rho(m)\right)^{2} .
$$

The pseudorandom weight we seek will turn out to be a scalar multiple of the above function: we defer the precise choice of normalization until the end of the proof of Proposition 5.

Lemma 3. When $\omega=c_{0} \log N$ and $R=N^{\eta}$ with $0<\eta \leqslant c_{0} / 2$, we have

$$
0 \leqslant \lambda_{b, W} \ll_{\eta} \Lambda_{\chi, R, W} \ll_{\varepsilon} N^{\varepsilon}
$$

for every $\varepsilon>0$.

Proof. If $\lambda_{b, W}(n)$ is non-zero, $W n+b$ is a prime of size at least $W>N^{c_{0} / 2}$, for $N$ large enough. Therefore any non-trivial divisor of $W n+b$ is larger than $R$, so that $\Lambda_{\chi, R, W}(n)=\frac{\phi(W)}{W}(\log R) \rho(1) \leqslant \eta^{-1} \lambda_{b, W}(n)$. The last inequality follows from standard bounds on the divisor function [32].

We now say more on the choice of weights $\rho(m)$. We let

$$
\rho(m)=\chi\left(\frac{\log m}{\log R}\right) \quad \text { where } \quad \chi(x)=1_{[-1,1]}(x) \cdot e^{x+1} e^{-1 /\left(1-x^{2}\right)}
$$


is the usual bump function multiplied by an exponential. By Fourier inversion, we may write $\chi(x)=\int_{-\infty}^{\infty} \varphi(\xi) e^{-(1+i \xi) x} \mathrm{~d} \xi$ for every $x \in[-1,1]$, where $\varphi$ is the Fourier transform of $1_{[-1,1]}(x) e^{1-1 /\left(1-x^{2}\right)}$, and thus decays as $^{6} \varphi(\xi) \ll e^{-c|\xi|^{1 / 2}}$ (see e.g. [16]). The interest in this choice is that, by truncation at a parameter $L \geqslant 1$, we may write

$$
\rho(m)=\int_{-L}^{L} m^{-(1+i \xi) / \log R} \varphi(\xi) d \xi+O\left(e^{-c L^{1 / 2}}\right) \quad(m \leqslant R) .
$$

This has the effect of introducing a small negative power of $m$ in the Euler products arising in computations, which simplifies their evaluation greatly.

We now begin the proof of Proposition 5. We fix $D \geqslant 1$ and $\omega=c_{0} \log N$, so that we may assume that $\omega$ is larger than any fixed constant depending on $D$. We then consider a system of affine-linear forms $\psi: \mathbb{Z}^{d} \rightarrow \mathbb{Z}^{t}$ of finite complexity such that $d, t,\|\dot{\psi}\| \leqslant D$. We let further implicit constants and explicit unsuscripted constants $c, C$ depend on $d, t,\|\dot{\psi}\|$, while subscripted constants $c_{0}, c_{1}, \ldots$ are absolute.

The first step of the proof is to unfold divisor sums in the correlation of divisor sums, and it is useful in this regard to introduce the notation $\Omega=[t] \times[2]$. Note also that the prime in $\sum^{\prime}$ means that the summation is restricted to square-free numbers. The following constitutes the beginning of the proof of [13, Theorem D.3], which we do not reproduce.

Proposition 6 (Unfolding sums). Given $\left(m_{i j}\right) \in \mathbb{N}^{\Omega}$, write $m_{i}=\left[m_{i 1}, m_{i 2}\right]$ and

$$
\alpha\left(m_{1}, \ldots, m_{t}\right)=\mathbb{P}_{n \in \mathbb{Z}_{m}^{d}}\left(m_{i} \mid W \psi_{i}(n)+b \quad \forall i \in[t]\right) .
$$

Let also $P \geqslant 1$. Then

$$
\begin{aligned}
& h_{R, W}^{-t} \sum_{n \in[P]^{d}} \Lambda_{\chi, R, W}\left[\psi_{1}(n)\right] \ldots \Lambda_{\chi, R, W}\left[\psi_{t}(n)\right] \\
= & P^{d} \cdot \sum_{\left(m_{i j}\right) \in \mathbb{N}^{\Omega}}^{\prime} \alpha\left(m_{1}, \ldots, m_{t}\right) \prod_{(i, j) \in \Omega} \mu\left(m_{i j}\right) \rho\left(m_{i j}\right)+O\left(R^{2|\Omega|} P^{d-1}\right)
\end{aligned}
$$

Before proceeding further, we analyze the function $\alpha$ appearing in Proposition 6. By the Chinese Remainder theorem, $\alpha\left(m_{1}, \ldots, m_{t}\right)$ is multiplicative in the variables $m_{i j}$, keeping in mind that $m_{i}=\left[m_{i 1}, m_{i 2}\right]$. Writing $m_{i j}=p^{r_{i j}}, r_{i}=\max \left(r_{i 1}, r_{i 2}\right)$, and $B=\left\{(i, j) \in \Omega: r_{i j}=1\right\}$, we have $r_{i}=1$ if and only if $r_{i j}=1$ for some $j \in[2]$, that is, if and only if the slice $B_{i}$ of $B$ at $i$ is non-empty. Therefore

$$
\alpha\left(p^{r_{1}}, \ldots, p^{r_{t}}\right)=\mathbb{P}_{n \in \mathbb{Z}_{p}^{d}}\left(p \mid W \psi_{i}(n)+b \quad \forall i: B_{i} \neq \varnothing\right)=: \alpha(p, B) .
$$

Motivated by this, we say that a non-empty set $B \subset \Omega$ is vertical when, for some $i \in[t]$, we have $B \subset\{i\} \times[2]$. We now estimate the size of the factors $\alpha(p, B)$.

${ }^{6}$ Using a weaker decay $\ll(1+|\xi|)^{-A}$ instead would yield a slightly weaker error term $(\log N)^{-1+\varepsilon}$ in Proposition 5. 
Proposition 7 (Local probabilities). For $B \neq \varnothing$, we have

$$
\alpha(p, B)= \begin{cases}0 & \text { if } p \leqslant \omega \\ p^{-1} & \text { if } p>\omega \text { and } B \text { is vertical } \\ O\left(p^{-2}\right) & \text { if } p>\omega \text { and } B \text { is not vertical }\end{cases}
$$

Proof. Recall that $\alpha(p, B)$ is defined by (5.3). When $p \leqslant \omega$, we have $p \mid W$ and $(b, W)=1$, therefore $p$ does not divide any value $W \psi_{i}(n)+b$ and $\alpha(p, B)=0$. When $p>\omega>\|\dot{\psi}\|$, we have $p \nmid W$ and $W \dot{\psi}_{i} \neq 0$ in $\mathbb{Z}_{p}$ for every $i \in[t]$. When $B$ is vertical, there is only one $i$ such that $B_{i}$ is non-empty and therefore $\alpha(p, B)=p^{-1}$. When $B$ is not vertical, there are at least two indices $i, j$ such that $B_{i}, B_{j} \neq \varnothing$. Since $p>\omega>2\|\dot{\psi}\|^{2}$, the linear forms $\dot{\psi}_{i}$ and $\dot{\psi}_{j}$ are linearly independent over $\mathbb{Z}_{p}$, and therefore $\alpha(p, B) \leqslant p^{-2}$.

For reasons that shall be clear in a moment, we define the following Euler factor.

Definition 7 (Euler factor). Let $\xi \in \mathbb{R}^{\Omega}$ and $z_{i j}=\left(1+i \xi_{i j}\right) / \log R$. We let

$$
E_{p, \xi}=\sum_{B \subset \Omega}(-1)^{|B|} \alpha(p, B) p^{-\sum_{(i, j) \in B} z_{i j}}
$$

The local estimates of Proposition 7 and the fact that $\operatorname{Re}\left(z_{i j}\right)>0$ ensure the absolute convergence of the product $\prod_{p} E_{p, \xi}$. We now return to the unfolded sum in Proposition 6 , in which we proceed to replace the weights $\rho(m)$ by their truncated Fourier expression (5.2). This step being again well described in [13, Appendix D], we do not include the proof here.

Proposition 8 (Unfolding integrals). Writing $m_{i}=\left[m_{i 1}, m_{i 2}\right]$, we have, for any $L \geqslant 1$,

$$
\begin{aligned}
& \sum_{\left(m_{i j}\right) \in \mathbb{N}^{\Omega}}^{\prime} \alpha\left(m_{1}, \ldots, m_{t}\right) \prod_{(i, j) \in \Omega} \mu\left(m_{i j}\right) \rho\left(m_{i j}\right) \\
= & \int_{[-L, L]^{\Omega}} \ldots \int_{p} \prod_{p, \xi} \prod_{(i, j) \in \Omega} \varphi\left(\xi_{i j}\right) \mathrm{d} \xi_{i j}+O\left(e^{-c L^{1 / 2}}(\log R)^{|\Omega|}\right) .
\end{aligned}
$$

With the estimates on local probabilities at hand, one can easily estimate the Euler product arising in Proposition 8.

Proposition 9 (Euler product estimate). Let $1 \leqslant L \leqslant \frac{c \log R}{\log \omega}$ be a parameter. For every $\xi \in[-L, L]^{\Omega}$, we have

$$
\prod_{p} E_{p, \xi}=\left(1+O\left(\frac{1}{\omega}+\frac{L \log \omega}{\log R}\right)\right) \cdot h_{R, W}^{-t} \cdot \prod_{B \text { vertical }}\left(\sum_{(i, j) \in B}\left(1+i \xi_{i j}\right)\right)^{-(-1)^{|B|}} .
$$


Proof. Note at the outset the useful identity $\sum_{B \text { vertical }}(-1)^{|B|}=-t$, and write $z_{i j}=$ $\left(1+i \xi_{i j}\right) / \log R$ as in Definition 7. By Proposition 7, we have

$$
\begin{aligned}
\prod_{p} E_{p, \xi} & =\prod_{p>\omega}\left(1+\sum_{B \text { vertical }}(-1)^{|B|} p^{-1-\sum_{B} z_{i j}}+O\left(p^{-2}\right)\right) \\
& =\left(1+O\left(\omega^{-1}\right)\right) \prod_{p>\omega} \prod_{B \text { vertical }}\left(1-p^{-1-\sum_{B} z_{i j}}\right)^{-(-1)^{|B|}}
\end{aligned}
$$

Since $p^{-z}=1+O\left(\frac{L \log p}{\log R}\right)$ for $p \leqslant \omega$ and $|z| \leqslant L / \log R$, we have further

$$
\prod_{p} E_{p, \xi}=\left(1+O\left(\omega^{-1}\right)\right) \prod_{p \leqslant \omega}\left(1-p^{-1}+O\left(\frac{L \log p}{p \log R}\right)\right)^{-t} \prod_{B \text { vertical }} \zeta\left(1+\sum_{B} z_{i j}\right)^{(-1)^{|B|}} .
$$

Using the fact that $\zeta(s)=\frac{1}{s-1}(1+O(|s-1|))$ for $\operatorname{Re}(s)>1$, it follows that

$$
\prod_{p} E_{p, \xi}=\left(1+O\left(\frac{1}{\omega}+\frac{L \log \omega}{\log R}+\frac{L}{\log R}\right)\right)\left(\frac{\phi(W)}{W}\right)^{-t} \prod_{B \text { vertical }}\left(\sum_{B} z_{i j}\right)^{-(-1)^{|B|}},
$$

which concludes the proof upon recalling that $z_{i j}=\left(1+i \xi_{i j}\right) / \log R$.

At this stage, the following sieve factors arise.

Definition 8 (Sieve factor). We let

$$
c_{\chi, 2}=\iint_{\mathbb{R}^{2}} \frac{(1+i \xi)\left(1+i \xi^{\prime}\right)}{2+i\left(\xi+\xi^{\prime}\right)} \varphi(\xi) \varphi\left(\xi^{\prime}\right) \mathrm{d} \xi \mathrm{d} \xi^{\prime} .
$$

The last step is to replace the euler product $\prod_{p} E_{p, \xi}$ in Proposition 8 by its approximation obtained in Proposition 9, and to extend the range of integration back to $\mathbb{R}$. Again, we refer to [13, Appendix D] for the proof of this familiar step.

Proposition 10 (Refolding integrals). Provided that $1 \leqslant L \leqslant \frac{c \log R}{\log \omega}$, we have

$$
h_{R, W}^{t} \int_{[-L, L]^{\Omega}} \ldots \int_{p} \prod_{p, \xi} \prod_{(i, j) \in \Omega} \varphi\left(\xi_{i j}\right) \mathrm{d} \xi_{i j}=c_{\chi, 2}^{t}+O\left(e^{-c L^{1 / 2}}+\frac{1}{\omega}+\frac{L \log \omega}{\log R}\right) .
$$

We also quote [13, Lemma D.2], which provides an explicit formula for $c_{\chi, 2}$.

Lemma 4. We have $c_{\chi, 2}=\int_{0}^{\infty}\left|\chi^{\prime}(x)\right|^{2} \mathrm{~d} x$.

We may now combine the previous successive approximations to the original sum and optimize the parameter $L$ to obtain Proposition 5 .

Proof of Proposition 5. Let $P \geqslant 1$. Combining Propositions 6, 8 and 10, we see that the average $\mathbb{E}_{n \in[P]^{d}} \prod_{i \in[t]} \Lambda_{\chi, R, W}\left[\psi_{i}(n)\right]$ is equal to

$$
c_{\chi, 2}^{t}+O\left(e^{-c L^{1 / 2}}(\log R)^{O(1)}+\frac{1}{\omega}+\frac{L \log \omega}{\log R}+\frac{R^{5 t}}{P}\right),
$$


provided that $L \leqslant \frac{c \log R}{\log \omega}$. Recall now that $\omega=c_{0} \log N$. Assuming that $P \geqslant N^{c_{1}}$, we choose $L=C(\log \log N)^{2}$ and $R=N^{c_{2} / t}$ for a small $c_{2}>0$, so that

$$
\mathbb{E}_{n \in[P]^{d}} \prod_{i \in[t]} \Lambda_{\chi, R, W}\left[\psi_{i}(n)\right]=c_{\chi, 2}^{t}+O\left((\log N)^{-1+o(1)}\right) .
$$

By Lemma 4, we have $c_{\chi, 2}>0$ and therefore we may define a renormalized weight $\nu:=c_{\chi, 2}^{-1} \Lambda_{\chi, R, W}$, which satisfies the desired pseudorandomness asymptotic by (5.5), and which majorizes a constant multiple of $\lambda_{b, W}$ by Lemma 3 .

\section{Quantitative PSEUdorandomness}

The goal of this section is to transfer the previous pseudorandomness asymptotics over $\mathbb{Z}$ to the setting of a large cyclic group, and to show that pseudorandomness is preserved under certain averaging operations. We also state the generalized Von Neumann theorem of Green and Tao [13, Appendix C], in a quantified form. The relevant notion of pseudorandomness in our paper is the following.

Definition 9 (Quantitative pseudorandomness). Let $D, H \geqslant 1$ be parameters and let $M$ be a prime. We say that $\nu: \mathbb{Z}_{M} \rightarrow \mathbb{R}^{+}$is D-pseudorandom of level $H$ when, for every affine system $\theta: \mathbb{Z}_{M}^{d} \rightarrow \mathbb{Z}_{M}^{t}$ of finite complexity such that $d, t,\|\dot{\theta}\| \leqslant D$,

$$
\mathbb{E}_{n \in \mathbb{Z}_{M}^{d}} \nu\left[\theta_{1}(n)\right] \ldots \nu\left[\theta_{t}(n)\right]=1+O_{D}\left(\frac{1}{H}\right) .
$$

We now let $N$ denote an integer larger than some absolute constant, and as in the previous section we fix $\omega=c_{0} \log N$ and $W=\prod_{p \leqslant \omega} p$. We also consider an embedding $[N] \hookrightarrow \mathbb{Z}_{M}$, where $M$ is a prime larger than $N$. We are then interested in finding a pseudorandom majorant over $\mathbb{Z}_{M}$ for the function $\lambda_{b, W}$ from Definition 5, properly extended to a function on $\mathbb{Z}_{M}$. Precisely, given a function $f: \mathbb{Z} \rightarrow \mathbb{C}$ with support in $[N]$, we define an $M$-periodic function $\widetilde{f}$ at $n \in \mathbb{Z}$ by $\widetilde{f}(n)=f(n+\ell M)$, where $\ell$ is the unique integer such that $n+\ell M \in[M]$, and that function $\tilde{f}$ may in turn be viewed as a function on $\mathbb{Z}_{M}$.

It is actually relatively simple to construct a pseudorandom majorant on $\mathbb{Z}_{M}$ from the one of Proposition 5 , by cutting $\mathbb{Z}_{M}^{d}$ into small boxes as explained in [12, p. 527]. We rerun this argument here since we need to extract explicit error terms from it.

Proposition 11 (Pseudorandom majorant over $\mathbb{Z}_{M}$ ). Let $D \geqslant 1$. There exists a constant $C_{D}$ such that if $N \geqslant C_{D}$ and $M \geqslant N$ is a prime, there exists a D-pseudorandom weight $\widetilde{\nu}: \mathbb{Z}_{M} \rightarrow \mathbb{R}^{+}$of level $(\log N)^{1-o(1)}$ such that

$$
0 \leqslant \widetilde{\lambda}_{b, W} \ll_{D} \widetilde{\nu}
$$

Proof. Consider an affine system $\theta: \mathbb{Z}_{M}^{d} \rightarrow \mathbb{Z}_{M}^{t}$ of finite complexity and such that $d, t,\|\dot{\theta}\| \leqslant D$. By Fact 2 , we may consider $\theta$ as the reduction modulo $M$ of an affine 
system $\psi: \mathbb{Z}^{d} \rightarrow \mathbb{Z}^{t}$ with norms $\|\psi\|_{M}=\|\theta\| \leqslant 2 D$ and $\|\dot{\psi}\|=\|\dot{\theta}\| \leqslant D$. We let further implicit constants depend on $D$ in the course of this proof.

Let $\nu$ be the weight from Proposition 5 , and define $\widetilde{\nu}: \mathbb{Z}_{M} \rightarrow \mathbb{R}^{+}$as above. Choosing another scale $P=M^{1 / 2}$, and duplicating the variable of averaging, we obtain

$$
\mathbb{E}_{n \in[M]^{d}} \prod_{i \in[t]} \widetilde{\nu}\left[\psi_{i}(n)\right]=\mathbb{E}_{m \in[M]^{d}} \mathbb{E}_{n \in[P]^{d}} \prod_{i \in[t]} \widetilde{\nu}\left[\psi_{i}(m+n)\right]+O\left(N^{-1 / 4}\right) .
$$

We call an integer $m$ good when $\psi\left(m+[P]^{d}\right) \subset[M]^{t}+M \ell$ for some $\ell \in \mathbb{Z}^{t}$, and when that is not the case we say that $m$ is bad. When $m$ is good we have, with $\ell \in \mathbb{Z}^{t}$ as prescribed and by (5.1),

$$
\begin{aligned}
\mathbb{E}_{n \in[P]^{d}} \prod_{i \in[t]} \widetilde{\nu}\left[\psi_{i}(m+n)\right] & =\mathbb{E}_{n \in[P]^{d}} \prod_{i \in[t]} \nu\left[\dot{\psi}_{i}(n)+\left(\psi_{i}(m)-M \ell_{i}\right)\right] \\
& =1+O_{D}\left((\log N)^{-1+o(1)}\right) .
\end{aligned}
$$

When $m$ is bad, we have $\min _{i \in[t]} d\left(\psi_{i}(m), M \mathbb{Z}\right) \leqslant\|\dot{\psi}\| P$ with respect to the canonical distance $d(x, y)=|x-y|$ on $\mathbb{R}$. Indeed, when that inequality does not hold, we have

$$
\left.\psi(m+] 0, P{ }^{d}\right) \cap\left\{y \in \mathbb{R}^{t}: \exists i \in[t] \text { such that } y_{i} \in M \mathbb{Z}\right\}=\varnothing,
$$

and since $\left.\psi(m+] 0, P{ }^{d}\right)$ is connected it must be contained in one of the boxes $] 0, M\left[{ }^{t}+M \ell\right.$, $\ell \in \mathbb{Z}^{t}$ (it is helpful to draw a picture at this point). We have thus proven that when $m$ is bad, there exists $i \in[t]$ and $\ell_{i} \in \mathbb{Z}$ such that $\psi_{i}(m) \in \ell_{i} M+[-O(P), O(P)]$, and such an $\ell_{i}$ is necessarily $\ll 1+\|\psi\|_{M} \ll 1$. It is easy to check that the number of such $m \in[M]^{d}$ is $\ll P M^{d-1}=M^{d-1 / 2}$. Inserting the estimate (6.2) on good-boxes averages in (6.1), and neglecting the count of bad-boxes averages, we obtain the desired asymptotic.

The notion of pseudorandomness is quite robust under averaging operations, as demonstrated by the following proposition, which is needed later on to majorize certain convolutions of $\lambda_{b, W}$.

Proposition 12. Let $D, H \geqslant 1$ be parameters and $M$ be a prime. Suppose that $\nu$ : $\mathbb{Z}_{M} \rightarrow \mathbb{R}^{+}$is D-pseudorandom of level $H, B$ is a symmetric subset of $\mathbb{Z}_{M}$ and $\mu_{B}=$ $(|B| / M)^{-1} 1_{B}$. Then $\nu^{\prime}=\frac{1}{2}\left(\nu+\nu * \mu_{B}\right)$ is also D-pseudorandom of level $H$.

Proof. Consider an affine system $\theta: \mathbb{Z}_{M}^{d} \rightarrow \mathbb{Z}_{M}^{t}$ of finite complexity such that $d, t,\|\dot{\theta}\| \leqslant$ $D$. Let $\nu^{(0)}=\nu$ and $\nu^{(1)}=\nu * \mu_{B}$, so that $\nu^{(\varepsilon)}(x)=\mathbb{E}_{y \in B} \nu(x+\varepsilon y)$ for every $\varepsilon \in\{0,1\}$ and $x \in \mathbb{Z}_{M}$. Therefore

$$
\begin{aligned}
S & :=\mathbb{E}_{n \in \mathbb{Z}_{M}^{d}} \frac{\nu^{(0)}+\nu^{(1)}}{2}\left[\theta_{1}(n)\right] \cdots \frac{\nu^{(0)}+\nu^{(1)}}{2}\left[\theta_{t}(n)\right] \\
& =\mathbb{E}_{\varepsilon \in\{0,1\}^{t}} \mathbb{E}_{n \in \mathbb{Z}_{M}^{d}} \nu^{\left(\varepsilon_{1}\right)}\left[\theta_{1}(n)\right] \cdots \nu^{\left(\varepsilon_{t}\right)}\left[\theta_{t}(n)\right] \\
& =\mathbb{E}_{\varepsilon \in\{0,1\}^{t}} \mathbb{E}_{y \in B^{t}} \mathbb{E}_{n \in \mathbb{Z}_{M}^{d}} \nu\left[\theta_{1}(n)+\varepsilon_{1} y_{1}\right] \cdots \nu\left[\theta_{t}(n)+\varepsilon_{t} y_{t}\right] .
\end{aligned}
$$


For every $\varepsilon \in\{0,1\}^{t}$ and $y \in B^{t}$, the system $\left(\theta_{i}+\varepsilon_{i} y_{i}\right)_{1 \leqslant i \leqslant t}$ has same linear part as $\left(\theta_{i}\right)_{1 \leqslant i \leqslant t}$. Since $\nu$ is $D$-pseudorandom of level $H$, we have $S=1+O_{D}\left(H^{-1}\right)$ as desired.

We now quote the generalized Von Neumann theorem of Green and Tao [13, Appen$\operatorname{dix} \mathrm{C}]$. It is simple to quantify the error term in that result in terms of the level of pseudorandomness of the weight.

Theorem 3 (Generalized Von Neumann theorem). Let $d, t, Q, H \geqslant 1$ and $s \geqslant 0$ be parameters, and let $i \in[t]$ be an indice. There exists a constant $D$ depending on $d, t, Q$ such that the following holds. Suppose that $M>D$ is a prime and $\theta: \mathbb{Z}_{M}^{d} \rightarrow \mathbb{Z}_{M}^{t}$ is an affine system of finite complexity in exact s-normal form at $i$, and such that $\|\dot{\theta}\| \leqslant Q$. Suppose also that $\nu: \mathbb{Z}_{M} \rightarrow \mathbb{R}^{+}$is D-pseudorandom of level $H$, and $f_{1}, \ldots, f_{t}: \mathbb{Z}_{M} \rightarrow \mathbb{R}$ are functions such that $\left|f_{j}\right| \leqslant \nu$ for every $j \in[t]$. Then we have

$$
\left|\mathbb{E}_{n \in \mathbb{Z}_{M}^{d}} f_{1}\left[\theta_{1}(n)\right] \cdots f_{t}\left[\theta_{t}(n)\right]\right|^{2^{s+1}} \leqslant\left\|f_{i}\right\|_{U^{s+1}\left(\mathbb{Z}_{M}\right)}^{2^{s+1}}+O_{D}\left(H^{-1}\right) .
$$

Proof. Up to relabeling the $f_{j}$ and $\theta_{j}$, we may assume that $i=1$. Up to permutating the base vectors, we may also assume that the set $J_{1}$ from Definition 2 is equal to $[s+1]$. It then suffices to apply [13, Proposition 7.1"], whose proof invokes twice the pseudorandomness condition of Definition 9, under the name "linear forms condition". Note that the argument there requires a change of variable $\left(x_{1}, \ldots, x_{s+1}, y\right) \mapsto$ $\left(c_{1}^{-1} x_{1}, \ldots, c_{s+1}^{-1} x_{s+1}, y\right)$ with respect to the decomposition $\mathbb{Z}_{M}^{d}=\mathbb{Z}_{M}^{s+1} \times \mathbb{Z}_{M}^{d-(s+1)}$, where $c_{k}=\dot{\theta}_{1}\left(e_{k}\right)$. The condition $M>D \geqslant\|\dot{\theta}\|$ ensures that this is possible, however the new forms involved may have large size, potentially not bounded in terms of $\|\dot{\theta}\|$. Fortunately, it can be verified that making the change of variables $x_{i} \mapsto c_{i} c_{s+1} x_{i}, 1 \leqslant i \leqslant s+1$ before each application of the linear forms condition in the proof of [13, Proposition 7.1"] converts the systems of forms under consideration back into sytems of bounded size. (Here we elaborated slightly on the footnote at the bottom of [13, p. 1822]).

\section{Translation-INVARIANT EQUATIONS IN THE PRIMES}

In this Section, we prove Theorem 2. Our two main tools are the transference principle of Helfgott and de Roton [14], including Naslund's [20] refinement thereof, and the relative generalized Von Neumann theorem of Green and Tao, in the quantitative form obtained in the previous section. These two tools together transfer the problem of finding a complexity-one pattern in the primes, to that of finding one in the integers, and to finish the proof we simply apply our extension of Shao's result derived in Appendix A.

We now formally begin the proof of Theorem 2. We start with a standard preliminary reduction, the $W$-trick, which allows us to consider subsets of an arithmetic progression of modulus $W$ in the primes instead. 
Theorem 4 (Theorem 2 in $W$-tricked primes). Let $V \in \mathcal{M}_{r \times t}(\mathbb{Z})$ be a translationinvariant matrix of rank $r$ and complexity one. There exists a constant $C$ depending at most on $r, t, V$ such that the following holds. Let $W=\prod_{p \leqslant \omega} p$, where $\omega=c_{0} \log N$ with $c_{0} \in\left[\frac{1}{4}, \frac{1}{2}\right]$, and let $b \in \mathbb{Z}$ such that $(b, W)=1$. Suppose that $A$ is a subset of $[N]$ such that $b+W \cdot A \subset \mathcal{P}$ and

$$
\begin{gathered}
|A|=\alpha(W / \phi(W))(\log N)^{-1} N, \\
\alpha \geqslant C(\log \log N)^{-1 / 25 t} .
\end{gathered}
$$

Then there exists $\mathbf{y} \in A^{t}$ with distinct coordinates such that $V \mathbf{y}=0$.

Proof that Theorem 4 implies Theorem 2.

Consider a subset $A$ of $\mathcal{P}_{N}$ of density $\alpha$; we may certainly assume that $\alpha \geqslant C N^{-1 / 4}$, and in particular that $N$ is large enough. Let $W=\prod_{p \leqslant \omega} p$, where $\omega=\frac{1}{4} \log N$, and let $N^{\prime}=\lfloor N / W\rfloor=N^{3 / 4+o(1)}$ (by the prime number theorem) be another scale. By [14, Lemma 2.1], there exists $(b, W)=1$ such that $A^{\prime}=\left\{n \in\left[N^{\prime}\right]: b+W n \in A\right\}$ has size $\gg \alpha(W / \phi(W))\left(\log N^{\prime}\right)^{-1} N^{\prime}$. Note that $\omega \sim \frac{1}{3} \log N^{\prime}$ as $N \rightarrow \infty$, and since $b+W \cdot A^{\prime} \subset$ $A$, every solution $\mathbf{y} \in\left(A^{\prime}\right)^{t}$ to $V \mathbf{y}=0$ with distinct coordinates induces one in $A^{t}$, by translation-invariance and homogeneity. Applying then Theorem 4 to $A^{\prime} \subset\left[N^{\prime}\right]$ concludes the proof.

From now on, we work under the hypotheses of Theorem 4. First, we fix a translationinvariant matrix $V \in \mathcal{M}_{r \times t}(\mathbb{Z})$ of complexity one, and without loss of generality we may assume that $t \geqslant 3$ and $V$ has no zero columns. Via Propositions 3 and 4 , we can choose a linear parametrization $\psi: \mathbb{Z}^{d} \rightarrow \mathbb{Z}^{t} \cap \operatorname{Ker}_{\mathbb{Q}}(V)$ in exact 1 -normal form over $\mathbb{Z}$ at every $i \in[t]$. We assume from now on that $N$ is large enough with respect to $d, t, \psi, V$, and we let further implicit and explicit constants depend on those parameters. We will need to consider functions with support in $[-2 N, 2 N]_{\mathbb{Z}}$, and to analyze those we embed $[-2 N, 2 N]_{\mathbb{Z}}$ in a large cyclic group $\mathbb{Z}_{M}$, where $M$ is a prime between $4(\|V\|+1) \cdot N$ and $8(\|V\|+1) \cdot N$ chosen via Bertrand's postulate. By Fact 1 , the linear map $\psi$ reduces modulo $M$ to a linear map $\theta: \mathbb{Z}_{M}^{d} \rightarrow \operatorname{Ker}_{\mathbb{Z}_{M}}(V)$ in exact 1-normal form over $\mathbb{Z}_{M}$ at every $i \in[t]$, and such that $\|\theta\|=\|\psi\|$; we work exclusively with that map from now on.

Next, we consider an integer $N \geqslant 1$ and a constant $c_{0} \in\left[\frac{1}{4}, \frac{1}{2}\right]$, and we fix

$$
W=\prod_{p \leqslant \omega} p, \quad \omega=c_{0} \log N, \quad b \in \mathbb{Z}:(b, W)=1 .
$$

We then consider a subset $A \subset[N]$ such that $|A|=\alpha \frac{W}{\phi(W)}(\log N)^{-1} \cdot N$ and $b+W \cdot A \subset \mathcal{P}$. Accordingly, we define the normalized indicator function of $A$ by

$$
\lambda_{A}=L \frac{\phi(W)}{W}(\log N) \cdot 1_{A}
$$


where $L=M / N \asymp 1$. With this normalization, we have $\mathbb{E}_{[M]} \lambda_{A}=\alpha$ and $0 \leqslant \lambda_{A} \ll \lambda_{b, W}$, recalling Definition 5 .

Given a function $f: \mathbb{Z} \rightarrow \mathbb{C}$ with support in $[-2 N, 2 N]$, we define an $M$-periodic function $\breve{f}(n)=0$ at $n \in \mathbb{Z}$ by $\breve{f}(n)=f(n+\ell M)$, where $\ell$ is the unique integer such that $n+\ell M \in[-M / 2, M / 2]_{\mathbb{Z}}$, and $\breve{f}$ may then be considered as a function on $\mathbb{Z}_{M}$. When $f$ has support in $[N]$, as is the case for $\lambda_{b, W}$, this coincides with the definition of $\tilde{f}$ from Section 6 . To alleviate the notation, we now identify functions $f: \mathbb{Z} \rightarrow \mathbb{C}$ with support in $[-2 N, 2 N]$ with their periodic counterpart $\breve{f}$. Most of the analysis we do next takes place on $\mathbb{Z}_{M}$, and Fourier transforms, convolutions, $L^{p}$ and $U^{k}$ norms are normalized accordingly. With these notations in place, we now work with the following pattern-counting operator.

Definition 10. We define the operator $T$ on functions $f_{1}, \ldots, f_{t}: \mathbb{Z}_{M} \rightarrow \mathbb{R}$ by

$$
T\left(f_{1}, \ldots, f_{t}\right)=\mathbb{E}_{n \in \mathbb{Z}_{M}^{d}} f_{1}\left[\theta_{1}(n)\right] \ldots f_{t}\left[\theta_{t}(n)\right] .
$$

If need be, we can always return to averages over $\mathbb{Z}$ via the following observation.

Lemma 5. For functions $f_{1}, \ldots, f_{t}: \mathbb{Z}_{M} \rightarrow \mathbb{R}$ with support in $[-2 N, 2 N]$, we have

$$
T\left(f_{1}, \ldots, f_{t}\right)=M^{-(t-r)} \sum_{\substack{y \in[-2 N, 2 N]_{\mathbb{Z}}^{t}: \\ V y=0}} f_{1}\left(y_{1}\right) \ldots f_{t}\left(y_{t}\right) .
$$

Proof. Since $\theta$ is a surjection onto $\operatorname{Ker}_{\mathbb{Z}_{M}}(V)$, and the fibers $\#\left\{x \in \mathbb{Z}_{M}^{d}: \theta(x)=y\right\}$ have uniform size when $y$ ranges over $\operatorname{Ker}_{\mathbb{Z}_{M}}(V)$, we have

$$
\begin{aligned}
T\left(f_{1}, \ldots, f_{t}\right) & =\mathbb{E}_{y \in \mathbb{Z}_{M}^{t}: V y=0} f_{1}\left(y_{1}\right) \ldots f_{t}\left(y_{t}\right) \\
& =M^{-(t-r)} \sum_{y \in \mathbb{Z}_{M}^{t}: V y=0} f_{1}\left(y_{1}\right) \ldots f_{t}\left(y_{t}\right) .
\end{aligned}
$$

Since the $f_{i}$ have support in $[-2 N, 2 N]$, we may restrict the summation to $y \in[-2 N, 2 N]_{\mathbb{Z}}^{t}$, and since $M>2\|V\| N$, the identity $V y=0$ holds in $\mathbb{Z}$ for such $y$.

We now introduce two parameters $\delta \in(0,1]$ and $\varepsilon \in(0, c]$. We also fix an auxiliary Bohr set of $\mathbb{Z}_{M}$ (see Definition 11) defined by

$$
\begin{aligned}
& \Gamma=\left\{r \in \mathbb{Z}_{M}:\left|\widehat{\lambda}_{A}(r)\right| \geqslant \delta\right\} \cup\{1\}, \\
& B=B(\Gamma, \varepsilon) .
\end{aligned}
$$

The presence of 1 in the frequency set guarantees that the Bohr set is contained in an interval $[-\varepsilon M, \varepsilon M]$. As is common in the transference literature for three-term arithmetic progressions $[9,10,14,20]$, we work with a smooth approximation of $\lambda_{A}$, namely the convolution over $\mathbb{Z}$ given by

$$
\lambda_{A}^{\prime}=\lambda_{A} * \lambda_{B},
$$


where $\lambda_{B}=|B|^{-1} 1_{B}$. Provided that $\varepsilon$ is small enough, we see that the support of $\lambda_{A}^{\prime}$ is contained in $[-2 N, 2 N]$. Since $M>2 N$, we may also consider $\lambda_{A}^{\prime}: \mathbb{Z}_{M} \rightarrow \mathbb{R}$ as the normalized convolution over $\mathbb{Z}_{M}$ given by

$$
\lambda_{A}^{\prime}=\lambda_{A} * \mu_{B}
$$

where $\mu_{B}=(|B| / M)^{-1} 1_{B}$. To show that $\lambda_{A}^{\prime}$ is close to $\lambda_{A}$ in a Fourier $\ell^{4}$ sense, we need to call on the restriction estimates of Green and Tao [10], themselves based on an envelopping sieve of Ramaré and Ruzsa [21]; these estimates were in turn specialized to the case of a large modulus $\omega$ by Helfgott and de Roton [14], and an alternative approach to those can be found in a blog post of Tao [28].

Proposition 13. We have $\left\|\lambda_{A}-\lambda_{A}^{\prime}\right\|_{U^{2}} \ll \varepsilon^{1 / 4}+\delta^{1 / 4}$.

Proof. By [14, Lemma 2.2], we have $\sum_{r}\left|\widehat{\lambda}_{A}(r)\right|^{q} \ll_{q} 1$ for any $q>2$. Therefore,

$$
\begin{aligned}
\left\|\lambda_{A}-\lambda_{A}^{\prime}\right\|_{U^{2}}^{4} & =\sum_{r}\left|\widehat{\lambda}_{A}(r)\right|^{4}\left|1-\widehat{\mu}_{B}(r)\right|^{4} \\
& \ll \varepsilon \sum_{r:\left|\widehat{\lambda}_{A}(r)\right| \geqslant \delta}\left|\widehat{\lambda}_{A}(r)\right|^{4}+\delta \sum_{r:\left|\widehat{\lambda}_{A}(r)\right| \leqslant \delta}\left|\widehat{\lambda}_{A}(r)\right|^{3} \\
& \ll \varepsilon+\delta,
\end{aligned}
$$

where we used the fact that $\left|1-\widehat{\mu}_{B}(r)\right|=\left|\mathbb{E}_{x \in B}\left(1-e_{N}(r x)\right)\right| \leqslant 2 \pi \varepsilon$ for all $r \in \Gamma$.

The structure of our argument is now as follows: we compare the counts $T\left(\lambda_{A}, \ldots, \lambda_{A}\right)$ and $T\left(\lambda_{A}^{\prime}, \ldots, \lambda_{A}^{\prime}\right)$, which we expect to be close by Proposition 13 and the heuristic that "the $U^{2}$ norm controls complexity one averages".

Remark 1 (Multilinear expansion). By multilinearity,

$$
T\left(\lambda_{A}, \ldots, \lambda_{A}\right)=T\left(\lambda_{A}^{\prime}, \ldots, \lambda_{A}^{\prime}\right)+\sum T\left(*, \ldots, \lambda_{A}-\lambda_{A}^{\prime}, \ldots, *\right) .
$$

where the sum is over $2^{t}-1$ terms and the stars stand for functions equal to $\lambda_{A}^{\prime}$ or $\lambda_{A}-\lambda_{A}^{\prime}$.

To estimate the main term in (7.2), that is, $T\left(\lambda_{A}^{\prime}, \ldots, \lambda_{A}^{\prime}\right)$, we invoke a key transference estimate of Helfgott and de Roton [14], which essentially allows us to consider $\lambda_{A}^{\prime}$ as a subset of the integers of density $\alpha^{2}$. It is further possible, by a result of Naslund ${ }^{7}$ [20], to obtain an exponent $1+o(1)$ instead of 2 , and we choose to work with that more efficient version, even though it is possible to derive Theorem 2 with a smaller exponent without it. This is because we wish to exhibit that our argument preserves the exponent in Szemerédi-type theorems in the integers, in the sense of Proposition 5 below.

${ }^{7}$ Here we implicitely refer to the first version of Naslund's preprint, because the argument there is simpler, and we do not seek very sharp bounds on the exponent. 
Proposition 14. Suppose that $\delta^{-4} \log \varepsilon^{-1} \leqslant c \log N$. Then for any $\kappa>0$, the level set $A^{\prime}=\left\{\lambda_{A}^{\prime} \geqslant \alpha / 2\right\}$ has density $\gg_{\kappa} \alpha^{1+\kappa}$ in $\mathbb{Z}_{M}$.

Proof. Recalling (7.1), we see that $\mathbb{E} \lambda_{A}^{\prime}=\mathbb{E} \lambda_{A}=\alpha$. By Selberg's sieve or the restriction estimate used in the proof of Proposition 13, we have

$$
\#\left\{r:\left|\widehat{\lambda}_{A}(r)\right| \geqslant \delta\right\} \leqslant \delta^{-4}\left\|\widehat{\lambda}_{A}\right\|_{4}^{4} \ll \delta^{-4},
$$

and therefore $|B| \geqslant \varepsilon^{|\Gamma|} N \geqslant N^{1 / 2}$ under our assumptions on $\varepsilon$ and $\delta$. By [20, Proposition 2], we deduce that $\left\|\lambda_{A}^{\prime}\right\|_{p} \ll_{p} 1$ for any even $p \geqslant 4$, and the proposition then follows from the $p$-th moment version of the classical Paley-Zygmund inequality.

Applying our statistical, complexity-one extension of Shao's result in the integers, we can now obtain a lower bound on the average of $\lambda_{A}^{\prime}$ over $\psi$-configurations.

Proposition 15 (Main term). Suppose that $\delta^{-4} \log \varepsilon^{-1} \leqslant c \log N$. We have

$$
T\left(\lambda_{A}^{\prime}, \ldots, \lambda_{A}^{\prime}\right) \geqslant \exp \left[-C_{\kappa} \alpha^{-24 t-\kappa}\right]
$$

for every $\kappa>0$.

Proof. Consider the level set $A^{\prime}=\left\{\lambda_{A}^{\prime} \geqslant \alpha / 2\right\}$ contained in the support of $\lambda_{A}^{\prime}$, and therefore in $[-2 N, 2 N]$. Since $\lambda_{A}^{\prime} \geqslant(\alpha / 2) \cdot 1_{A^{\prime}}$, we have

$$
T\left(\lambda_{A}^{\prime}, \ldots, \lambda_{A}^{\prime}\right) \geqslant(\alpha / 2)^{t} T\left(1_{A^{\prime}}, \ldots, 1_{A^{\prime}}\right) .
$$

By Proposition 14, we know that $A^{\prime}$ has density $\gg_{\kappa} \alpha^{1+\kappa}$ in $[-2 N, 2 N]$ for any $\kappa>0$. Invoking Lemma 5, and applying Proposition 17 to $A^{\prime} \subset[-2 N, 2 N]$, we obtain

$$
T\left(1_{A^{\prime}}, \ldots, 1_{A^{\prime}}\right)=M^{-(t-r)} \#\left\{y \in\left(A^{\prime}\right)^{t}: V y=0\right\} \geqslant \exp \left[-C_{\kappa} \alpha^{-(1+\kappa) 24 t}\right] .
$$

On the other hand, the averages from (7.2) involving a difference $\lambda_{A}-\lambda_{A}^{\prime}$ are bounded via the generalized Von Neumann theorem of Section 6.

Proposition 16 (Error terms). Suppose that $f_{1}, \ldots, f_{t}$ are functions all equal to $\lambda_{A}^{\prime}$ or $\lambda_{A}-\lambda_{A}^{\prime}$, with at least one of them equal to $\lambda_{A}-\lambda_{A}^{\prime}$. Then

$$
\left|T\left(f_{1}, \ldots, f_{t}\right)\right| \ll \varepsilon^{1 / 4}+\delta^{1 / 4}+(\log N)^{-\frac{1}{4}+o(1)} .
$$

Proof. We consider $i \in[t]$ such that $f_{i}=\lambda_{A}-\lambda_{A}^{\prime}$. Let $Q=\|\dot{\theta}\|$ and let $D=D_{d, t, Q}$ be the constant from Proposition 3. By Proposition 11, and since we assumed $N$ to be large enough with respect to $d, t, \theta$, there exists a $D$-pseudorandom weight $\nu: \mathbb{Z}_{M} \rightarrow \mathbb{R}^{+}$ of level $(\log N)^{1-o(1)}$ such that

$$
0 \leqslant \lambda_{A} \ll \lambda_{b, W} \ll \nu
$$


Let $\nu^{\prime}=\frac{1}{2}\left(\nu+\nu * \mu_{B}\right)$, so that $\left|\lambda_{A}^{\prime}\right| \ll \nu^{\prime}$ and $\left|\lambda_{A}-\lambda_{A}^{\prime}\right| \ll \nu^{\prime}$. By Proposition $12, \nu^{\prime}$ is also $D$-pseudorandom of level $(\log N)^{1-o(1)}$.

Recall now that $\psi$ is in exact 1-normal form at $i$. Applying Proposition 3 with $s=1$ to the functions $f_{1}, \ldots, f_{t}$ (divided by a certain large constant), and inserting the estimates of Proposition 13, we obtain the desired bound.

At this point we need only collect together the bounds on the main term and the error terms in (7.2) to finish the proof of Theorem 2, which we have previously reduced to proving Theorem 4.

Proof of Theorem 4. Starting from the multilinear expansion (7.2), and inserting the bounds from Propositions 15 and 16, we obtain

$$
T\left(\lambda_{A}, \ldots, \lambda_{A}\right) \geqslant \exp \left[-C_{\kappa} \alpha^{-24 t-\kappa}\right]-O\left(\varepsilon^{1 / 4}+\delta^{1 / 4}+(\log N)^{-\frac{1}{4}+o(1)}\right),
$$

whenever, say, $\varepsilon^{-1}, \delta^{-1} \leqslant c(\log N)^{1 / 8}$. Choose now $\varepsilon=\delta=\exp \left[-C_{\kappa}^{\prime} \alpha^{-24 t-\kappa}\right]$ (for a large $\left.C_{\kappa}^{\prime}\right)$, and assume that $\alpha \geqslant C_{\kappa}(\log \log N)^{-1 /(24 t+\kappa)}$. This ensures that the conditions on $\varepsilon$ and $\delta$ are satisfied, and that we have a lower bound

$$
T\left(\lambda_{A}, \ldots, \lambda_{A}\right) \geqslant \exp \left[-C_{\kappa}^{\prime} \alpha^{-24 t-\kappa}\right] .
$$

By Lemma 5 and since $\lambda_{A} \leqslant(\log N) 1_{A}$, we then have

$$
\#\left\{y \in A^{t}: V y=0\right\} \geqslant \exp \left[-C_{\kappa} \alpha^{-24 t-\kappa}\right] \cdot N^{t-r}(\log N)^{-t} .
$$

On the other hand, by Lemma 2, the number of $y \in[N]^{t}$ with two identical coordinates and such that $V y=0$ is $\ll N^{t-r-1}$. Choosing now $\kappa=t$ for aesthetic reasons, and given the range of density under consideration, we are therefore ensured to find at least one non-trivial solution.

As claimed before, our argument allows for a slightly more general statement than Theorem 2. Indeed, the following can be obtained by a suitable Varnavides argument and by inserting the resulting analog of Proposition 17 in our proof.

Theorem 5. Suppose that $V \in \mathcal{M}_{r \times t}(\mathbb{Z})$ is a translation-invariant matrix of rank $r$ and complexity one, and let $\gamma>0$ be a parameter. Assume that $V \mathbf{y}=0$ has a distinctcoordinates solution $\mathbf{y} \in A^{t}$ for every subset $A$ of $[N]$ of density at least

$$
C(\log N)^{-\gamma}
$$

Then such a solution also exists for every subset $A$ of $\mathcal{P}_{N}$ of density at least

$$
C_{\varepsilon}(\log \log N)^{-\gamma+\varepsilon},
$$

for any $\varepsilon>0$. 
This being said, we have not tried to optimize the exponent $1 / 24 t$ in Corollary 1 , nor the exponent in Theorem 2 that follows from it. This is because the former exponent is likely not optimal, and far from comparable in quality with Sanders' [24] bounds for Roth's theorem, because of the repeated applications of Cauchy-Schwarz in Appendix A.

\section{Appendix A. Translation-INVARIAnt EQUATIONS IN THE INTEGERS}

The purpose of this section is to derive an extension of a result of Shao [26] to arbitrary systems of complexity one, and with a count of the multiplicity of pattern occurences. The structure of our proof is similar to Shao's, and it relies in particular in the key local inverse $U^{2}$ theorem proved there (Proposition 21 below). However, certain added technicalities arise when handling arbitrary systems: the most significant of those is addressed by Proposition 20 below.

Proposition 17. Let $V \in \mathcal{M}_{r \times t}(\mathbb{Z})$ be a translation-invariant matrix of rank $r$ and complexity one. Suppose that $A$ is a subset of $[-N, N]_{\mathbb{Z}}$ of density $\alpha$. Then

$$
\#\left\{\mathbf{y} \in A^{t}: V \mathbf{y}=0\right\} \geqslant \exp \left[-C \alpha^{-24 t}\right] \cdot N^{t-r},
$$

for a constant $C>0$ depending at most on $r, t, V$.

Although we only need the result above for the transference argument of Section 7 , we record the following consequence, since it may be of independent interest.

Corollary 1. Let $V \in \mathcal{M}_{r \times t}(\mathbb{Z})$ be a translation-invariant matrix of rank $r$ and complexity one. There exists a constant $C>0$ depending at most on $r, t, V$ such that, if $A$ is a subset of $[N]$ of density at least $C(\log N)^{-1 / 24 t}$, there exists a solution $\mathbf{y} \in A^{t}$ to $V \mathbf{y}=0$ with distinct coordinates.

Proof. By Lemma 2, the number of $\mathbf{y} \in[N]^{t}$ with two equal coordinates such that $V \mathbf{y}=0$ is at most $O\left(N^{t-r-1}\right)$. The result then follows from Proposition 17, since we assumed that $\alpha \geqslant C(\log N)^{-1 / 24 t}$.

We now fix a translation-invariant matrix $V \in \mathcal{M}_{r \times t}(\mathbb{Z})$ of rank $r$, and for the purpose of proving Proposition 17, we may assume without loss of generality that $t \geqslant 3$ and $V$ has no zero columns. By Propositions 3 and 4, we may choose a linear parametrization $\varphi: \mathbb{Z}^{q+1} \rightarrow \mathbb{Z}^{t} \cap \operatorname{Ker}_{\mathbb{Q}}(V)$ of the form $\varphi\left(x_{0}, x\right)=x_{0} \mathbf{1}+\psi(x)$, where $\psi: \mathbb{Z}^{q} \rightarrow \mathbb{Z}^{t}$ is in exact 1-normal form at every $i \in[t]$. We have traded the letter $d$ for $q$ here because the former is too precious as the dimension of a Bohr set. Writing $\psi_{i}(x)=a_{i 1} x_{1}+\cdots+a_{i q} x_{q}$, we define the sets of non-zero coefficients $\Xi_{i}=\left\{a_{i j} \neq 0, j \in[q]\right\}$ and $\Xi=\cup_{i \in[t]} \Xi_{i}$, so that we have $|a| \leqslant\|\varphi\|$ for every $a \in \Xi$.

We also consider a fixed integer $N$ from the statement of Proposition 17, which should be thought of as quite large. As usual, we choose to carry out our Fourier analysis over 
a cyclic group $\mathbb{Z}_{M}$ on a slightly larger scale; to be precise, via Bertrand's postulate we pick a prime $M$ such that $\|\varphi\| \cdot 2 N<M \leqslant\|\varphi\| \cdot 4 N$. Finally, throughout this section the letters $c$ and $C$ denote positive constants which are chosen, respectively, small or large enough with respect to $q, t$ and $\varphi$. While we do not attempt to track the dependency of our parameters on $\|\varphi\|$, we sometimes use this quantity to illustrate our argument.

We now recall the basics of Bohr sets and regularity calculus, which can be found in many places $[8,11,15]$. We speed up this process as this material is utterly standard and our notation is consistent with the litterature.

Definition 11. A Bohr set of frequency set $\Gamma \subset \mathbb{Z}_{M}$ and radius $\delta>0$ is

$$
B(\Gamma, \delta)=\left\{x \in \mathbb{Z}_{M}:\left\|\frac{x r}{M}\right\| \leqslant \delta \quad \forall r \in \Gamma\right\}
$$

and its dimensiond is defined by $d=|\Gamma|$. We often let the parameters $\Gamma, \delta, d$ be implicitely defined whenever we introduce a Bohr set $B$. The $\rho$-dilate $B_{\mid \rho}$ of a Bohr set $B$ is defined by $B(\Gamma, \delta)_{\mid \rho}=B(\Gamma, \rho \delta)$, and given two Bohr sets $B, B^{\prime}$ we write $B^{\prime} \leqslant{ }_{\rho} B$ when $B^{\prime} \subset B_{\mid \rho}$. Finally, we say that $B$ is regular when, for every $0<\rho \leqslant 2^{-6} / d$,

$$
\left(1-2^{6} \rho d\right)|B| \leqslant\left|B_{\mid 1 \pm \rho}\right| \leqslant\left(1+2^{6} \rho d\right)|B| .
$$

We also recall standard size estimates on Bohr sets, as well as Bourgain's regularization lemma. In our later argument, all Bohr sets will be picked regular.

Fact 3. Suppose that $B$ is a Bohr set of dimension $d$ and radius $\delta$, and $\rho \in(0,1]$. Then

$$
|B| \geqslant \delta^{d} M \quad \text { and } \quad\left|B_{|\rho|}\right| \geqslant(\rho / 2)^{2 d}|B|
$$

Given any Bohr set $B$, there exists $c \in\left[\frac{1}{2}, 1\right]$ such that $B_{\mid c}$ is regular.

In practice, regularity is used in the following form, close in spirit to [11, Lemma 4.2]. When we argue "by regularity" in a proof, we implicitely invoke these estimates.

Fact 4 (Regularity calculus). Let $f: \mathbb{Z}_{M} \rightarrow[-1,1]$ and suppose that $B$ is a regular $d$-dimensional Bohr set, $X^{\prime} \subset B_{\mid \rho}$ is another set and $x^{\prime} \in B_{\mid \rho}$, where $\rho \in(0, c / d]$. Then

$$
\begin{aligned}
\mathbb{E}_{x \in x^{\prime}+B} f(x) & =\mathbb{E}_{x \in B} f(x)+O(\rho d), \\
\mathbb{E}_{x \in B} f(x) & =\mathbb{E}_{x \in B, x^{\prime} \in X^{\prime}} f\left(x+x^{\prime}\right)+O(\rho d), \\
\mathbb{E}_{x \in B} 1\left(x \in B_{\mid 1-\rho}\right) f(x) & =\mathbb{E}_{x \in B} f(x)+O(\rho d) .
\end{aligned}
$$

Before proceeding further, we recall certain facts about Gowers box norms [13, Appendix B], which are present in disguise in Shao's argument [26]. For our argument, we only require the positivity of such norms, and two Cauchy-Schwarz-based inequalities. Strictly speaking, we could do without those norms, however they are useful to write averages over cubes in a more compact (if less intuitive) form, and to expedite 
repeated applications of Cauchy-Schwarz. In the following definitions, we let $X_{1}, X_{2}$ denote arbitrary subsets of $\mathbb{Z}_{M}$.

Definition 12 (Box scalar product and norm). The box scalar product of a family of functions $\left(h_{\omega}: X_{1} \times X_{2} \rightarrow \mathbb{R}\right)_{\omega \in\{0,1\}^{2}}$ is

$$
\left\langle\left(h_{\omega}\right)\right\rangle_{\square\left(X_{1} \times X_{2}\right)}=\mathbb{E}_{x^{(0)}, x^{(1)} \in X_{1} \times X_{2}} \prod_{\omega \in\{0,1\}^{2}} h_{\omega}\left(x_{1}^{\left(\omega_{1}\right)}, x_{2}^{\left(\omega_{2}\right)}\right) .
$$

The box norm of a function $h: X_{1} \times X_{2} \rightarrow \mathbb{R}$ is defined by $\|h\|_{\square\left(X_{1} \times X_{2}\right)}^{4}=\langle(h)\rangle_{\square\left(X_{1} \times X_{2}\right)}$.

The first inequality we require is a box Van der Corput inequality implicit in [7, p. 161], while the second is the Gowers-Cauchy-Schwarz inequality [13, Lemma B.2].

Fact 5. For $h: X_{1} \times X_{2} \rightarrow \mathbb{R}$ and $\left(b_{k}: X_{k} \rightarrow[-1,1]\right)_{k \in\{1,2\}}$, we have

$$
\left|\mathbb{E}_{x_{1} \in X_{1}, x_{2} \in X_{2}} h\left(x_{1}, x_{2}\right) b_{1}\left(x_{1}\right) b_{2}\left(x_{2}\right)\right| \leqslant\|h\|_{\square\left(X_{1} \times X_{2}\right)} .
$$

For $\left(h_{\omega}: X_{1} \times X_{2} \rightarrow \mathbb{R}\right)_{\omega \in\{0,1\}^{2}}$, we have

$$
\left|\left\langle\left(h_{\omega}\right)\right\rangle_{\square\left(X_{1} \times X_{2}\right)}\right| \leqslant \prod_{\omega \in\{0,1\}^{2}}\left\|h_{\omega}\right\|_{\square\left(X_{1} \times X_{2}\right)} .
$$

In our situation, we need a slight variant of the local $U^{2}$ norm defined in [26].

Definition 13 (Twisted $U^{2}$ norm). Let $a, b \in \mathbb{Z}$ and $g: \mathbb{Z}_{M} \rightarrow \mathbb{R}$. The $(a, b)$-twisted $U^{2}$ norm of $g$ with respect to $X_{1}, X_{2}$ is

$$
\|g\|_{\bigotimes_{a, b}\left(X_{1} \times X_{2}\right)}^{4}=\mathbb{E}_{x^{(0)}, x^{(1)} \in X_{1} \times X_{2}} \prod_{\omega \in\{0,1\}^{2}} g\left(a x_{1}^{\left(\omega_{1}\right)}+b x_{2}^{\left(\omega_{2}\right)}\right) .
$$

When $a=b=1$ we simply write $\|g\|_{\otimes\left(X_{1} \times X_{2}\right)}$.

With these notations, the local Gowers norm of a function $f$ with respect to sets $X_{0}, X_{1}, X_{2}$ as defined by Shao [26, Definition 3.1] is

$$
\|f\|_{U^{2}\left(X_{0}, X_{1}, X_{2}\right)}^{4}=\mathbb{E}_{x_{0} \in X_{0}}\left\|f\left(x_{0}+\cdot\right)\right\|_{\bigotimes\left(X_{1} \times X_{2}\right)}^{4} .
$$

From now on we keep the suggestive "local Gowers norm" terminology, but we use the expression in the right-hand side for computational purposes.

We are now ready to start with the proof of Proposition 17. We introduce, for a system of Bohr sets $\mathbf{B}=\left(B_{0}, \ldots, B_{q}\right)$, the multilinear operator on functions

$$
T_{\mathbf{B}}\left(f_{1}, \ldots, f_{t}\right)=\mathbb{E}_{x_{0} \in B_{0}, \ldots, x_{q} \in B_{q}} f_{1}\left[\varphi_{1}(x)\right] \ldots f_{t}\left[\varphi_{t}(x)\right] .
$$

The next proposition then constitutes the first step of our density increment strategy, in which we deduce that a set $A$ either possesses many $\varphi$-configurations, or it induces a large $T_{\mathbf{B}}$-average involving the balanced function of $A$. Here and in the following, 
we occasionally make superfluous assumptions on the Bohr sets involved, in order to facilitate the combination of intermediate propositions.

Proposition 18 (Multilinear expansion). Suppose that $A$ is a subset of density $\alpha$ of a regular d-dimensional Bohr set $B=B_{0}$, and write $f_{A}=1_{A}-\alpha 1_{B}$. Suppose also that $B_{1}, \ldots, B_{q}$ are regular Bohr sets with $B_{i} \leqslant \rho B_{i-1}$ for all $i \in[q]$, where $\rho \leqslant c / d$. Then either

(i) (Many patterns) $T_{\mathbf{B}}\left(1_{A}, \ldots, 1_{A}\right) \geqslant \alpha^{t} / 4$,

(ii) (Large $T$-average) or there exist functions $f_{1}, \ldots, f_{t}: \mathbb{Z}_{M} \rightarrow[-1,1]$ and $i \in[t]$ such that $f_{i}=f_{A}$ and $\left|T_{\mathbf{B}}\left(f_{1}, \ldots, f_{t}\right)\right| \gg \alpha^{t}$.

Proof. First observe that, expanding $1_{A}=\alpha 1_{B}+f_{A}$ by multilinearity,

$$
T_{\mathbf{B}}\left(1_{A}, \ldots, 1_{A}\right)=T_{\mathbf{B}}\left(\alpha 1_{B}, \ldots, \alpha 1_{B}\right)+\sum T_{\mathbf{B}}\left(*, \ldots, f_{A}, \ldots, *\right)
$$

where the sum is over $2^{t}-1$ terms and the stars stand for functions equal to $\alpha 1_{B}$ or $f_{A}$. By definition,

$$
T_{\mathbf{B}}\left(\alpha 1_{B}, \ldots, \alpha 1_{B}\right)=\alpha^{t} \mathbb{E}_{x_{0} \in B} \mathbb{E}_{x \in B_{1} \times \cdots \times B_{q}} 1_{B}\left[x_{0}+\psi_{1}(x)\right] \ldots 1_{B}\left[x_{0}+\psi_{t}(x)\right] .
$$

Restricting $x_{0}$ to lie in $B_{\mid 1-\rho}$ with $\rho \leqslant c /\|\varphi\| d$, we are ensured that $x_{0}+\psi_{j}(x) \in B$ for every $j \in[t]$ and $x \in B_{1} \times \cdots \times B_{q} \subset B_{\mid \rho}^{q}$. By regularity, we thus have

$$
\begin{aligned}
T_{\mathbf{B}}\left(\alpha 1_{B}, \ldots, \alpha 1_{B}\right) & =\alpha^{t}\left(\mathbb{E}_{x_{0} \in B} 1_{B_{\mid 1-\rho}}\left(x_{0}\right)+O(\rho d)\right) \\
& =(1+O(\rho d)) \alpha^{t} \\
& \geqslant \alpha^{t} / 2 .
\end{aligned}
$$

By (A.3), if we are not in the first case of the proposition, then by the pigeonhole principle there must exist a large average

$$
\alpha^{t} \ll\left|T_{\mathbf{B}}\left(f_{1}, f_{2}, \ldots, f_{t}\right)\right|
$$

where one of the functions $f_{i}: \mathbb{Z}_{M} \rightarrow[-1,1]$ is equal to $f_{A}$.

The next step is to use the fact that (twisted) local Gowers norms control the count of $\varphi$-configurations, up to a small error. This is the analog for general systems of complexity 1 of Shao's [26, Proposition 4.1]; it is also very similar to Green and Tao's generalized Von Neumann theorem for bounded functions [7, Theorem 2.3].

Proposition 19 (Large average implies large Gowers norm). Let $\eta \in(0,1]$ be a parameter, and suppose that $B_{0}, \ldots, B_{q}$ are regular d-dimensional Bohr sets such that $B_{i} \leqslant{ }_{\rho} B_{i-1}$ for all $i \in[q]$, where $\rho \leqslant c \eta^{4} / d$. Suppose that $f_{1}, \ldots, f_{t}: \mathbb{Z}_{M} \rightarrow[-1,1]$ are such that

$$
\left|T_{\mathbf{B}}\left(f_{1}, \ldots, f_{t}\right)\right| \geqslant \eta .
$$


Then for every $i \in[t]$, there exist $1 \leqslant k<\ell \leqslant q$ and $a, b \in \Xi_{i}$ such that

$$
\mathbb{E}_{u_{0} \in B_{0}}\left\|f_{i}\left(u_{0}+\cdot\right)\right\|_{\bigotimes_{a, b}\left(B_{k} \times B_{\ell}\right)}^{4} \geqslant \eta / 2
$$

Proof. Let $i \in[t]$, and recall that $\psi$ is in exact 1-normal form at $i$. We may therefore find indices $1 \leqslant k<\ell \leqslant q$ and a partition $[t] \backslash\{i\}=X_{k} \sqcup X_{\ell}$ into non-empty sets such that $\psi_{i}$ depends on the variables $x_{k}$ and $x_{\ell}$, while for $j \in X_{k}$ (respectively $j \in X_{\ell}$ ), $\psi_{j}$ depends at most on the variable $x_{k}$ (respectively $x_{\ell}$ ) among those two variables. We decompose vectors $x \in \mathbb{Z}^{q+1}$ accordingly as $x=\left(x_{0}, x_{k}, x_{\ell}, y\right)$ with $y \in \prod_{j \notin\{0, k, l\}} B_{j}$, and we may write $\psi_{i}\left(x_{k}, x_{\ell}, y\right)=a_{k} x_{k}+a_{\ell} x_{\ell}+\psi_{i}(0,0, y)$ with $a_{k}, a_{\ell} \in \Xi_{i}$. Then ${ }^{8}$

$$
\begin{aligned}
\eta \leqslant \mid \mathbb{E}_{x_{0} \in B_{0}, y \in\left(B_{j}\right)_{j \notin\{0, k, \ell\}}} \mathbb{E}_{x_{k} \in B_{k}, x_{\ell} \in B_{\ell}} f_{i}\left[x_{0}+\psi_{i}\left(x_{k}, x_{\ell}, y\right)\right] \\
\times \prod_{j \in X_{k}} f_{j}\left[x_{0}+\psi_{j}\left(x_{k}, y\right)\right] \prod_{j \in X_{\ell}} f_{j}\left[x_{0}+\psi_{j}\left(x_{\ell}, y\right)\right] \mid .
\end{aligned}
$$

We may rewrite the averaged function as $h\left(x_{k}, x_{\ell}\right) b_{k}\left(x_{k}\right) b_{\ell}\left(x_{\ell}\right)$, where $h, b_{k}, b_{\ell}$ are functions depending on $x_{0}, y$ and $b_{k}, b_{\ell}$ are bounded by 1 . By Hölder's inequality, followed by the box Van der Corput inequality (A.1), we thus have

$$
\begin{aligned}
\eta^{4} & \leqslant\left(\mathbb{E}_{x_{0} \in B_{0}, y \in\left(B_{j}\right)_{j \notin\{0, k, \ell\}}}\left|\mathbb{E}_{x_{k} \in B_{k}, x_{\ell} \in B_{\ell}} h\left(x_{k}, x_{\ell}\right) b_{k}\left(x_{k}\right) b_{\ell}\left(x_{\ell}\right)\right|\right)^{4} \\
& \leqslant \mathbb{E}_{x_{0} \in B^{(0)}, y \in\left(B_{j}\right)_{j \notin\{0, k, \ell\}}}\left|\mathbb{E}_{x_{k} \in B_{k}, x_{\ell} \in B_{\ell}} h\left(x_{k}, x_{\ell}\right) b_{k}\left(x_{k}\right) b_{\ell}\left(x_{\ell}\right)\right|^{4} \\
& \leqslant \mathbb{E}_{x_{0} \in B_{0}, y \in\left(B_{j}\right)_{j \notin\{0, k, \ell\}}}\|h\|_{\square\left(B_{k} \times B_{\ell}\right)}^{4} .
\end{aligned}
$$

Unfolding the definition of the box norm, and by regularity on the variable $x_{0}$, we have

$$
\begin{gathered}
\eta^{4} \leqslant \mathbb{E}_{x_{0} \in B_{0}, y \in\left(B_{j}\right)_{j \notin\{0, k, \ell\}}} \mathbb{E}_{x^{(0)}, x^{(1)} \in B_{k} \times B_{\ell}} \\
\prod_{\omega \in\{0,1\}^{2}} f_{i}\left(x_{0}+a_{k} x_{k}^{\left(\omega_{k}\right)}+a_{\ell} x_{\ell}^{\left(\omega_{\ell}\right)}+\psi_{i}(0,0, y)\right) \\
=\mathbb{E}_{x_{0} \in B_{0}} \mathbb{E}_{x^{(0)}, x^{(1)} \in B_{k} \times B_{\ell}} \prod_{\omega \in\{0,1\}^{2}} f_{i}\left(x_{0}+a_{k} x_{k}^{\left(\omega_{k}\right)}+a_{\ell} x_{\ell}^{\left(\omega_{\ell}\right)}\right)+O(\rho d) .
\end{gathered}
$$

Refolding the definition of the $\left(a_{k}, a_{\ell}\right)$-twisted $U^{2}$ norm, this concludes the proof, provided that $\rho \leqslant c \eta^{4} / d$.

We now wish to reduce the conclusion of the previous proposition to the situation where $a=b=1$, that is, when $f_{A}$ has a large (regular) local Gowers norm. It turns out that such a reduction is always possible by a simple averaging argument, together with an application of the Gowers-Cauchy-Schwarz inequality to separate the translated functions arising from such a process.

Proposition 20. Let $\eta \in(0,1]$ be a parameter. Suppose that $B_{0}, B_{1}, B_{2}$ are regular d-dimensional Bohr sets such that $B_{1}, B_{2} \leqslant \rho B_{0}$, and consider two other Bohr sets

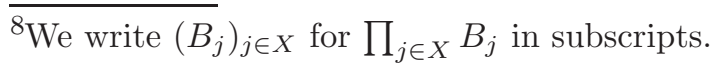


$\widetilde{B}_{1} \leqslant \widetilde{\rho} B_{1}$ and $\widetilde{B}_{2} \leqslant \widetilde{\rho} B_{2}$, where $\rho, \widetilde{\rho} \leqslant c \eta^{4} / d$. Then for $f: \mathbb{Z}_{M} \rightarrow[-1,1]$ and $a, b \in \Xi$,

$$
\mathbb{E}_{u_{0} \in B_{0}}\left\|f\left(u_{0}+\cdot\right)\right\|_{\bigotimes_{a, b}\left(B_{1} \times B_{2}\right)}^{4} \geqslant \eta^{4} \Rightarrow \mathbb{E}_{u_{0} \in B_{0}}\left\|f\left(u_{0}+a b \cdot\right)\right\|_{\square\left(\widetilde{B}_{1} \times \widetilde{B}_{2}\right)}^{4} \geqslant \eta^{4} / 2
$$

Proof. Unfolding the definition of the twisted $U^{2}$ norm, we have

$$
\eta^{4} \leqslant \mathbb{E}_{u_{0} \in B_{0}} \mathbb{E}_{x^{(0)}, x^{(1)} \in B_{1} \times B_{2}} \prod_{\omega \in\{0,1\}^{2}} f\left(u_{0}+a x_{1}^{\left(\omega_{1}\right)}+b x_{2}^{\left(\omega_{2}\right)}\right) .
$$

By regularity, we now duplicate the variables $x_{1}^{(\varepsilon)}$ into $x_{1}^{(\varepsilon)}+b y_{1}^{(\varepsilon)}$ with $y_{1}^{(\varepsilon)} \in \widetilde{B}_{1}$, and the variables $x_{2}^{(\varepsilon)}$ into $x_{2}^{(\varepsilon)}+a y_{2}^{(\varepsilon)}$ with $y_{2}^{(\varepsilon)} \in \widetilde{B}_{2}$, so that

$$
\begin{aligned}
\eta^{4}-O(\widetilde{\rho} d) \leqslant \mathbb{E}_{u_{0} \in B_{0}} \mathbb{E}_{x^{(0)}, x^{(1)} \in B_{1} \times B_{2}} \mathbb{E}_{y^{(0)}, y^{(1)} \in \widetilde{B}_{1} \times \widetilde{B}_{2}} & \prod_{\omega \in\{0,1\}^{2}} f\left(u_{0}+a x_{1}^{\left(\omega_{1}\right)}+b x_{2}^{\left(\omega_{2}\right)}+a b\left(y_{1}^{\left(\omega_{1}\right)}+y_{2}^{\left(\omega_{2}\right)}\right)\right) \\
= & \mathbb{E}_{u_{0} \in B_{0}} \mathbb{E}_{x^{(0)}, x^{(1)} \in B_{1} \times B_{2}}\left\langle\left(f\left(u_{0}+a x_{1}^{\left(\omega_{1}\right)}+b x_{2}^{\left(\omega_{2}\right)}+a b S\right)\right)_{\omega}\right\rangle_{\square\left(\widetilde{B}_{1} \times \widetilde{B}_{2}\right)},
\end{aligned}
$$

where $S: \widetilde{B}_{1} \times \widetilde{B}_{2} \rightarrow \mathbb{Z}_{M}$ is defined by $S\left(u_{1}, u_{2}\right)=u_{1}+u_{2}$. Applying successively the Gowers-Cauchy-Schwarz inequality (A.2) and Hölder's inequality, we obtain

$$
\begin{aligned}
c \eta^{16} & \leqslant\left(\mathbb{E}_{u_{0} \in B_{0}} \mathbb{E}_{x^{(0)}, x^{(1)} \in B_{1} \times B_{2}} \prod_{\omega \in\{0,1\}^{2}}\left\|f\left(u_{0}+a x_{1}^{\left(\omega_{1}\right)}+b x_{2}^{\left(\omega_{2}\right)}+a b S\right)\right\|_{\square\left(\widetilde{B}_{1} \times \widetilde{B}_{2}\right)}\right)^{4} \\
& \leqslant \prod_{\omega \in\{0,1\}^{2}} \mathbb{E}_{u_{0} \in B_{0}} \mathbb{E}_{x^{(0)}, x^{(1)} \in B_{1} \times B_{2}}\left\|f\left(u_{0}+a x_{1}^{\left(\omega_{1}\right)}+b x_{2}^{\left(\omega_{2}\right)}+a b S\right)\right\|_{\square\left(\widetilde{B}_{1} \times \widetilde{B}_{2}\right)}^{4} .
\end{aligned}
$$

By the pigeonhole principle, we may therefore find $\omega \in\{0,1\}^{2}$ such that

$$
\begin{aligned}
c \eta^{4} & \leqslant \mathbb{E}_{u_{0} \in B_{0}} \mathbb{E}_{x^{(0)}, x^{(1)} \in B_{1} \times B_{2}}\left\|f\left(u_{0}+a x_{1}^{\left(\omega_{1}\right)}+b x_{2}^{\left(\omega_{2}\right)}+a b S\right)\right\|_{\square\left(\widetilde{B}_{1} \times \widetilde{B}_{2}\right)}^{4} \\
& =\mathbb{E}_{u_{0} \in B_{0}}\left\|f\left(u_{0}+a b S\right)\right\|_{\square\left(\widetilde{B}_{1} \times \widetilde{B}_{2}\right)}^{4}+O(\rho d),
\end{aligned}
$$

where we have used regularity in the variable $u_{0}$ in the last step. The proposition follows from recalling Definition 13.

At this point, we have reduced to a situation where we may apply Shao's local inverse $U^{2}$ theorem [26, Theorem 3.2 and Lemma 5.1], quoted below, to obtain a density increment. The presence of a coefficient $m=a b$ calls for a minor variant ${ }^{9}$ of that result, which can however be effortlessly extracted out of Shao's argument: we omit the proof. Note also that in the proposition below, we consider Bohr sets of $\mathbb{Z}_{M}$ as sets of integers via the pullback of $\pi:[-M / 2, M / 2]_{\mathbb{Z}} \stackrel{\sim}{\rightarrow} \mathbb{Z}_{M}$.

Proposition 21 (Local inverse $U^{2}$ theorem [26]). Let $\eta \in\left(0, \frac{1}{2}\right]$ and $m \in \Xi \cdot \Xi$ be parameters. Suppose that $B_{0}, B_{1}, B_{2}$ are regulard-dimensional Bohr sets such that $B_{1} \leqslant \rho$

${ }^{9}$ Note also that Bohr sets on $\mathbb{Z}$ are used in that reference, however this is only a cosmetic difference. We actually quote a slightly weaker, but simpler, one-case consequence of Shao's result to fluidify our argument. 
$B_{0}$ and $B_{2} \leqslant \rho B_{1}$, where $\rho \leqslant c \eta^{12} / d$. Suppose also that $f: \mathbb{Z}_{M} \rightarrow[-1,1]$ is such that $\mathbb{E}_{B_{0}} f=0$ and

$$
\mathbb{E}_{u_{0} \in B_{0}}\left\|f\left(u_{0}+m \cdot\right)\right\|_{\unrhd\left(B_{1} \times B_{2}\right)}^{4} \gg \eta^{4} .
$$

Then there exists $u \in \mathbb{Z}$ and a regular Bohr set $B_{3}$ such that $u+m B_{3} \subset B_{0}$ in $\mathbb{Z}$, and

$$
d_{3} \leqslant d+1, \quad \delta_{3} \geqslant(\eta / d)^{O(1)} \delta_{1}, \quad \mathbb{E}_{u+m B_{3}} f \geqslant c \eta^{12} .
$$

We are now ready to combine the previous propositions into our main density-increment statement, which we then iterate to obtain Proposition 17.

Proposition 22 (Main iterative proposition). Suppose that $A$ is a subset of density $\alpha \in\left(0, \frac{1}{2}\right]$ of a regular d-dimensional Bohr set $B$ contained in $[-N, N]$. Then either

(i) (Many $\varphi$-configurations) we have

$$
\#\left\{x \in[-N, N]^{q+1}: \varphi(x) \in A^{t}\right\} \geqslant(\alpha \delta / d)^{O(d)} N^{q+1},
$$

(ii) (Density increment) or there exists $u \in \mathbb{Z}, m \in \mathbb{N}$ and a regular Bohr set $B^{\prime}$ such that $u+m B^{\prime} \subset B$ in $\mathbb{Z}$ and, writing $\alpha^{\prime}=\left|A \cap\left(u+m B^{\prime}\right)\right| /\left|B^{\prime}\right|$,

$$
\alpha^{\prime} \geqslant\left(1+c \alpha^{12 t-1}\right) \alpha, \quad d^{\prime} \leqslant d+1, \quad \delta^{\prime} \geqslant(\alpha / d)^{O(1)} \delta .
$$

Proof. Write $\eta=\alpha^{t}$ and choose $\rho=c \eta^{12} / d$. Let $B_{0}=B$, and choose regular Bohr sets $B_{1}, \ldots, B_{q}$ with $B_{i}=B_{i-1 \mid \rho_{i}}$ and $\rho_{i} \in[\rho / 2, \rho]$, so as to apply Proposition 18 . Since $B_{i} \subset[-N, N]$ and $M>2\|\varphi\| N$, for any $x \in B_{0} \times \cdots \times B_{q}, \varphi(x)$ belongs to $A^{t}$ modulo $M$ if and only if it does in $\mathbb{Z}$. Therefore, if we are in the first case of Proposition 18, we have

$$
\#\left\{x \in[-N, N]^{q+1}: \varphi(x) \in A^{t}\right\} \geqslant c \alpha^{t}\left|B_{0}\right| \ldots\left|B_{q}\right| \geqslant(\alpha \delta / d)^{O(d)} M^{q+1} .
$$

In the second case, we deduce, by Proposition 19, that there exist $i \in[t], 1 \leqslant k<\ell \leqslant q$ and twists $a, b \in \Xi_{i}$ such that, for $f_{A}=1_{A}-\alpha 1_{B_{0}}$,

$$
\mathbb{E}_{u_{0} \in B_{0}}\left\|f_{A}\left(u_{0}+\cdot\right)\right\|_{\bigotimes_{a, b}\left(B_{k} \times B_{\ell}\right)}^{4} \gg \eta^{4} .
$$

Via Proposition 20, we may assume instead that

$$
\mathbb{E}_{u_{0} \in B_{0}}\left\|f_{A}\left(u_{0}+a b \cdot\right)\right\|_{\bigotimes\left(\widetilde{B}_{k} \times \widetilde{B}_{\ell}\right)}^{4} \gg \eta^{4}
$$

for regular dilates $\widetilde{B}_{k}=B_{k \mid \rho_{k}}$ and $\widetilde{B}_{\ell}=B_{\ell \mid \rho_{\ell}}$ with $\rho_{k}, \rho_{\ell} \in[\rho / 2, \rho]$; note that we have $\widetilde{B}_{k} \leqslant_{2 \rho} \widetilde{B}_{\ell}$. Finally, an application of Proposition 21 to $f_{A}$ yields a density increment of the desired shape.

Proof of Proposition 17. As stated at the beginning of this section, we use a parametrization $\varphi: \mathbb{Z}^{q+1} \rightarrow \mathbb{Z}^{t} \cap \operatorname{Ker}_{\mathbb{Q}}(V)$, so that $\operatorname{rk}(\varphi)=\operatorname{dim}\left(\operatorname{Ker}_{\mathbb{Q}} V\right)=t-r$. We embed $[-N, N]$ in a regular Bohr set $B^{(0)}:=B\left(\{1\}, \frac{c}{D}\right)$ of $\mathbb{Z}_{M}$, where $c \in[1,2]$ and $M=D N$. The 
set $A^{(0)}:=A$ then has density $\gg \alpha$ in $B^{(0)}$. We now construct iteratively a sequence of regular Bohr sets $B^{(i)}$ of dimension $d_{i}$ and radius $\delta_{i}$ contained in $[-N, N]$, and a sequence of subsets $A_{i}$ of $B^{(i)}$ of density $\alpha_{i}$; we also view $A_{i}$ as subsets of $\mathbb{Z}$ via the pullback of $\pi:[-M / 2, M / 2]_{\mathbb{Z}} \stackrel{\sim}{\longrightarrow} \mathbb{Z}_{M}$. At each step we apply Proposition 22 to the set $A_{i}$, and in the second case of that proposition we define $A_{i+1}$ in $\mathbb{Z}$ by

$$
A_{i} \cap\left(u_{i+1}+m_{i+1} B_{i+1}\right)=u_{i+1}+m_{i+1} A_{i+1} .
$$

Writing $S_{\varphi}(Y)=\#\left\{x \in[-N, N]^{q+1}: \varphi(x) \in Y^{t}\right\}$ for a set of integers $Y$, it follows from the linearity and the presence of a shift variable in $\varphi$ that $S_{\varphi}(A) \geqslant S_{\varphi}\left(A_{i}\right)$ for every $i$.

From $\alpha_{i+1} \geqslant\left(1+c \alpha_{i}^{12 t-1}\right) \alpha_{i}$ and a familiar geometric series summation [8, Chapter 6], we deduce that the algorithm runs for at most $O\left(\alpha^{-12 t+1}\right)$ steps. Iterating the dimension and radius bounds, we also deduce that $d_{i} \ll \alpha^{-12 t+1}$ and $\delta_{i} \geqslant \exp \left[-C \alpha^{-12 t+1} \log \alpha^{-1}\right]$. Bounding crudely $\alpha^{2} \log \alpha^{-1} \ll 1$, we have therefore, in the first case of Proposition 22,

$$
\#\left\{x \in[-N, N]^{q+1}: \varphi(x) \in A^{t}\right\} \geqslant \exp \left[-C \alpha^{-24 t}\right] \cdot N^{q+1} .
$$

Since $\varphi$ has rank $t-r$, for each $y \in[N]^{t}$, we have the multiplicity bound

$$
\#\left\{x \in[-N, N]^{q+1}: \varphi(x)=y\right\} \ll N^{(q+1)-(t-r)} .
$$

Summing over values $y=\varphi(x)$ in (A.5), we have therefore

$$
\#\left\{y \in A^{t}: V y=0\right\} \geqslant \exp \left[-C \alpha^{-24 t}\right] \cdot N^{t-r} .
$$

\section{REFERENCES}

1. J. Bourgain, On triples in arithmetic progression, Geom. Funct. Anal. 9 (1999), no. 5, 968-984.

2. D. Conlon, J. Fox, and Y. Zhao, The Green-Tao theorem, an exposition, Preprint (2014), http://arxiv.org/abs/1403.2957.

3. J. Dousse, On a generalisation of Roth's theorem for arithmetic progressions and applications to sum-free subsets, Math. Proc. Cambridge Philos. Soc. 155 (2013), no. 2, 331-341.

4. D. A. Goldston, J. Pintz, and C. Y. Ylldırm, Primes in tuples. I, Ann. of Math. (2) 170 (2009), no. 2, 819-862.

5. D. A. Goldston and C. Y. Yıldırım, Higher correlations of divisor sums related to primes. III. Small gaps between primes, Proc. Lond. Math. Soc. (3) 95 (2007), no. 3, 653-686.

6. W. T. Gowers, A new proof of Szemerédi's theorem, Geom. Funct. Anal. 11 (2001), no. 3, 465-588.

7. W. T. Gowers and J. Wolf, The true complexity of a system of linear equations, Proc. Lond. Math. Soc. (3) 100 (2010), no. 1, 155-176.

8. A. Granville and B. Green, Additive combinatorics, Upcoming book (2014).

9. B. Green, Roth's theorem in the primes, Ann. of Math. (2) 161 (2005), no. 3, 1609-1636.

10. B. Green and T. Tao, Restriction theory of the Selberg sieve, with applications, J. Théor. Nombres Bordeaux 18 (2006), no. 1, 147-182. 
11. _ An inverse theorem for the Gowers $U^{3}(G)$ norm, Proc. Edinb. Math. Soc. (2) 51 (2008), no. $1,73-153$.

12. _ The primes contain arbitrarily long arithmetic progressions, Ann. of Math. (2) 167 (2008), no. $2,481-547$.

13. _ Linear equations in primes, Ann. of Math. (2) 171 (2010), no. 3, 1753-1850.

14. H. A. Helfgott and A. de Roton, Improving Roth's theorem in the primes, Int. Math. Res. Not. IMRN (2011), no. 4, 767-783.

15. K. Henriot, Bourgain's bounds for Roth's theorem, Expository note (2013), http://dms . umontreal.ca/ henriot/.

16. S. Johnson, Saddle-point integration of $C^{\infty}$ bump functions, Expository note (2006), http://math.mit.edu/ stevenj/.

17. T. H. Le and J. Wolf, Polynomial configurations in the primes, To appear in Int. Math. Res. Not. (2013).

18. Y.-R. Liu, C. V. Spencer, and X. Zhao, Roth's theorem on systems of linear forms in function fields, Acta Arith. 142 (2010), no. 4, 377-386.

19. _ A generalization of Meshulam's theorem on subsets of finite abelian groups with no 3-term arithmetic progression (II), European J. Combin. 32 (2011), no. 2, 258-264.

20. E. Naslund, On improving Roth's theorem in the primes, To appear in Mathematika (2014), First arxiv version: http://arxiv.org/abs/1302.2299v1, Second arxiv version: http://arxiv.org/abs/1302.2299.

21. O. Ramaré and I. Z. Ruzsa, Additive properties of dense subsets of sifted sequences, J. Théor. Nombres Bordeaux 13 (2001), no. 2, 559-581.

22. K. F. Roth, On certain sets of integers. II, J. London Math. Soc. 29 (1954), 20-26.

23. I. Z. Ruzsa, Solving a linear equation in a set of integers. I, Acta Arith. 65 (1993), no. 3, 259-282.

24. T. Sanders, On Roth's theorem on progressions, Ann. of Math. (2) 174 (2011), no. 1, 619-636.

25. T. Schoen and I. D. Shkredov, Roth's theorem in many variables, Preprint (2011), http://arxiv.org/abs/1106.1601.

26. X. Shao, Finding linear patterns of complexity one, To appear in Int. Math. Res. Not. IMRN (2013), http://arxiv.org/abs/1309.0644.

27. A. Shapira, Behrend-type constructions for sets of linear equations, Acta Arith. 122 (2006), no. 1, $17-33$.

28. T. Tao, Montgomery's uncertainty principle, Blog post (2011), http://terrytao . wordpress . com/2011/12/31/montgomerys-uncertainty-principle/.

29. _ Notes on linear patterns, Blog post (2010), http://terrytao.wordpress.com/.

30. T. Tao and T. Ziegler, The primes contain arbitrarily long polynomial progressions, Acta Math. 201 (2008), no. 2, 213-305.

31. _ Erratum to "The primes contain arbitrarily long polynomial progressions", Acta Math. 210 (2013), no. 2, 403-404.

32. G. Tenenbaum, Introduction to analytic and probabilistic number theory, Cambridge Studies in Advanced Mathematics, vol. 46, Cambridge University Press, Cambridge, 1995.

33. C. Vinuesa, Asymptotics for magic squares of primes, Preprint (2012), http://arxiv.org/abs/1207.3936. 
Département de mathématiques et de statistique, Université de Montréal, CP 6128 succ. Centre-Ville, Montréal QC H3C 3J7, Canada

Email address: henriot@dms.umontreal.ca 